\title{
The narrow Fe $\mathrm{K} \alpha$ line and the molecular torus in active galactic nuclei: an IR/X-ray view ${ }^{\star}$
}

\author{
C. Ricci ${ }^{1,2}$, Y. Ueda ${ }^{2}$, K. Ichikawa ${ }^{2}$, S. Paltani ${ }^{1}$, R. Boissay ${ }^{1}$, P. Gandhi ${ }^{3}$, M. Stalevski ${ }^{4,5,6,7}$, and H. Awaki ${ }^{8}$ \\ 1 Department of Astronomy, University of Geneva, ch. d'Ecogia 16, 1290 Versoix, Switzerland \\ 2 Department of Astronomy, Kyoto University, Oiwake-cho, Sakyo-ku, 606-8502 Kyoto, Japan \\ e-mail: ricci@kusastro.kyoto-u.ac.jp \\ 3 Department of Physics, University of Durham, South Road, Durham DH1 3LE, UK \\ 4 Astronomical Observatory, Volgina 7, 11060 Belgrade, Serbia \\ 5 Isaac Newton Institute of Chile, Yugoslavia Branch, Volgina 7, 11060 Belgrade, Serbia \\ ${ }^{6}$ Sterrenkundig Observatorium, Universiteit Gent, Krijgslaan 281-S9, 9000 Gent, Belgium \\ 7 Departamento de Astronomía, Universidad de Chile, Casilla 36-D, Correo Central, Santiago, Chile \\ 8 Department of Physics, Ehime University, 790-8577 Matsuyama, Japan
}

Received 18 September 2013 / Accepted 24 March 2014

\begin{abstract}
The narrow component of the iron $\mathrm{K} \alpha$ is an almost ubiquitous feature in the X-ray spectra of active galactic nuclei (AGN) and is believed to originate in neutral material, possibly located in the molecular torus. This would imply a tight connection between the $\mathrm{Fe} \mathrm{K} \alpha$ equivalent width (EW) and the physical properties of the torus. In a recent work we have shown that the decrease in the covering factor of the torus with the luminosity, as expected by luminosity-dependent unification models, would be able to explain the decrease in $\mathrm{Fe} \mathrm{K} \alpha \mathrm{EW}$ with the luminosity (i.e. the X-ray Baldwin effect). Recent developments in the study of the mid-IR (MIR) spectrum of AGN allow important parameters of the torus to be deduced, such as its covering factor $\left(f_{\text {obs }}\right)$ and equatorial column density $\left(N_{\mathrm{H}}^{\mathrm{T}}\right)$, by applying clumpy torus models. Using XMM-Newton/EPIC observations of a sample of 24 type-I AGN, we investigate the relation between the physical parameters of the torus obtained by recent MIR works and the properties of the Fe K $\alpha$ line. We correct the values of the $\mathrm{Fe} \mathrm{K} \alpha \mathrm{EW}$ by taking the inclination angle, the photon index, the equatorial column density, and half-opening angle of the torus into account using a physical torus model of X-ray reprocessed radiation. We find that the relation between Fe K $\alpha$ EW and $f_{\text {obs }}$ shows a slope that is consistent with the expected value, albeit with low statistical significance. A trend that is consistent with the theoretical prediction is also found when comparing the $\mathrm{Fe} \mathrm{K} \alpha \mathrm{EW}$ to $N_{\mathrm{H}}^{\mathrm{T}}$. Our work seems to confirm that the bulk of the narrow Fe K $\alpha$ line is produced by the same material responsible for the MIR emission.
\end{abstract}

Key words. galaxies: Seyfert - quasars: emission lines - quasars: general - X-rays: galaxies - infrared: galaxies - galaxies: active

\section{Introduction}

The unification model of active galactic nuclei (AGN) predicts that the supermassive black hole (SMBH) in their centre is surrounded by a molecular toroidal-like structure (Antonucci 1993). Anisotropic obscuration was originally required to explain the detection of broad lines in polarised light found in the optical spectrum of the Seyfert 2 NGC 1068 (Antonucci \& Miller 1985; Miller \& Antonucci 1983), and is now considered to be one of the fundamental ingredients needed to explain the structure of AGN. According to this paradigm Seyfert 1s (Sy1s) are observed pole-on with respect to the molecular torus, while Seyfert $2 \mathrm{~s}$ (Sy2s) are seen edge-on. The radiation produced by the central engine and absorbed by the torus is mainly re-emitted in the mid-IR band (MIR, 5-30 $\mu \mathrm{m}$ ). The first direct observation of the dusty torus was carried out using MIR interferometry, for NGC 1068 (Jaffe et al. 2004). This work was then followed by several others (e.g. Prieto et al. 2004, 2005; Meisenheimer et al. 2007; Tristram et al. 2007; Raban et al. 2009), and all of them detected a clear compact structure within few parsecs from the SMBH.

\footnotetext{
* Appendices are available in electronic form at http://www . aanda.org
}

The fluorescent iron $\mathrm{K} \alpha$ line is possibly the most important tracer of the material surrounding the SMBH. The Fe K $\alpha$ line is made of two components, $\mathrm{K} \alpha_{1}(E=6.404 \mathrm{keV})$ and $\mathrm{K} \alpha_{2}$ $(E=6.391 \mathrm{keV})$, with a branching ratio of $\mathrm{K} \alpha_{1}: \mathrm{K} \alpha_{2}=2: 1$, and it is produced when one of the two K-shell electrons of an iron atom is ejected following photoelectric absorption of an X-ray photon. After the photoelectric event, the excited state can decay in two ways. i) An L-shell electron drops into the K-shell releasing a photon. ii) The excess energy is carried away through the ejection of an L-shell electron (Auger effect). The fluorescent yield $(Y)$ determines the probability of fluorescence versus the Auger effect. The iron line is the strongest X-ray line produced from the reprocessing of the primary continuum, because of the Fe relative abundance, and because the fluorescent yield is proportional to the fourth power of atomic number $\left(Y \propto Z^{4}\right)$.

Amongst the other lines produced by $\mathrm{X}$-ray reflection from neutral material the strongest are the iron $\mathrm{K} \beta$ line at $7.06 \mathrm{keV}$ ( $\sim 13.5 \%$ of the flux of the $\mathrm{Fe} \mathrm{K} \alpha$, Palmeri et al. 2003), and the nickel $\mathrm{K} \alpha$ line at $\sim 7.47 \mathrm{keV}$ (e.g. Yaqoob \& Murphy 2011). The first evidence of an $\mathrm{Fe} \mathrm{K} \alpha$ line in the X-ray spectrum of an AGN was found by Mushotzky et al. (1978) when studying OSO-8 observations of Centaurus A. Ten years later Guilbert \& Rees (1988) and Lightman \& White (1988) predicted that fluorescent emission from neutral iron should be common in the X-ray 
spectra of Seyfert galaxies. Following this, Nandra et al. (1989) found evidence of an emission line at $E \sim 6 \mathrm{keV}$ in the EXOSAT spectrum of MCG-6 - 30 - 15. In the same year, using a larger sample Pounds et al. (1989) found significant iron $\mathrm{K} \alpha$ emission lines in the spectra of three more Seyfert 1 galaxies: NGC 5548, NGC 5506, and NGC 3227. Since then, thanks to the enormous progress in the development of X-ray detectors, iron lines have been found to be almost ubiquitous in AGN (e.g. Fukazawa et al. 2011).

The Fe $\mathrm{K} \alpha$ line is made of two components. While the narrow core of the line, with a full width at half maximum (FWHM) of $\simeq 2000 \mathrm{~km} \mathrm{~s}^{-1}$ (Shu et al. 2011), is observed in almost all AGN, in $\simeq 35-45 \%$ of the cases (de La Calle Pérez et al. 2010), an additional broadened component due to relativistic effects (e.g. Fabian \& Vaughan 2003) or to distortion of the continuum caused by clumpy ionised absorbers in the line-of-sight (e.g. Turner \& Miller 2009; Miyakawa et al. 2012) is found. The size of the narrow $\mathrm{Fe} \mathrm{K} \alpha$ emitting region is on average $\sim 3$ times larger than that of the broad line region (Shu et al. 2011), which seems to point towards most of the narrow core originating in the molecular torus. Another argument in favour of this scenario is the weak variability of reflection-dominated Compton-thick (CT, $N_{\mathrm{H}} \geq 10^{24} \mathrm{~cm}^{-2}$ ) AGN (e.g. Bianchi et al. 2012, and references therein). A torus origin of the $\mathrm{Fe} \mathrm{K} \alpha$ line would imply that its equivalent width (EW) is directly linked to the half-opening angle of the torus $\theta_{\mathrm{OA}}$ (Krolik et al. 1994) and to its equatorial column density $N_{\mathrm{H}}^{\mathrm{T}}$ (e.g. Ikeda et al. 2009; Murphy \& Yaqoob 2009).

An anti-correlation between the equivalent width of the $\mathrm{Fe} \mathrm{K} \alpha$ line and the X-ray luminosity of AGN has been found by a large number of studies of type-I AGN (e.g. Iwasawa \& Taniguchi 1993; Bianchi et al. 2007; Shu et al. 2010), and recently also in type-II AGN (Ricci et al. 2014). Such a trend is known as the X-ray Baldwin effect, for analogy with the Baldwin effect (Baldwin 1977), i.e. the decrease in the CIV $\lambda 1549 \mathrm{EW}$ with the luminosity. Several explanations have been proposed for the X-ray Baldwin effect: i) a luminosity-dependent variation in the ionisation state of the iron-emitting material (Nandra et al. 1997; Nayakshin 2000); ii) the effect of the delay between the primary X-ray emission and the reflection component (Jiang et al. 2006; Shu et al. 2012); iii) the decrease in the number of continuum photons in the iron line region with the Eddington ratio ( $\lambda_{\text {Edd }}$, Ricci et al. $2013 b$ ), as an effect of the correlation between the photon index $(\Gamma)$ of the continuum and $\lambda_{\text {Edd }}$ (e.g. Shemmer et al. 2008); iv) the decrease in the covering factor of the torus with the luminosity (e.g. Page et al. 2004; Zhou \& Wang 2005), as expected by luminosity-dependent unification models (e.g. Ueda et al. 2003). In a recent paper (Ricci et al. 2013a), we have shown that the decrease in the covering factor of the torus with the luminosity is able to reproduce the slope of the X-ray Baldwin effect for a wide range of equatorial column densities of the torus.

The thermal MIR continuum is produced by circumnuclear dust (e.g. Stalevski et al. 2012), which is heated by the optical/UV/X-ray photons produced in the disk and in the warm corona. The first attempts to model the MIR spectral energy distribution (SED) of AGN using a torus with a smooth density distribution (e.g. Pier \& Krolik 1992, 1993) were able to model part of the SED but not to produce realistic MIR spectra. This was probably the first evidence that dust in the torus does not have a smooth distribution. Already years earlier, Krolik \& Begelman (1988) hypothesised that the torus is made of optically thick dusty clouds, because a smooth distribution could not survive close to the SMBH. Several other pieces of evidence of a clumpy structure of the torus have been added in past years. From interferometric observations of Circinus galaxy Tristram et al. (2007) found that the data could not support a smooth-distribution scenario, but rather pointed towards the dust having a clumpy or filamentary structure. The discovery that Seyfert $1 \mathrm{~s}$ and Seyfert $2 \mathrm{~s}$ follow the same X-ray/MIR luminosity correlation (e.g. Gandhi et al. 2009; Ichikawa et al. 2012), and the detection of silicate emission in Seyfert 2s (Sturm et al. 2006) also provide strong arguments for the clumpy scenario. Important information about the structure of the torus (e.g. its covering factor and number of clouds, see Sect. 2) can be obtained by modelling the IR spectra of AGN using clumpy torus models such as the one developed by Nenkova et al. (2002). In the past few years, several studies (e.g. Mor et al. 2009; Alonso-Herrero et al. 2011) have carried out detailed analyses of the MIR properties of AGN for a significant number of objects.

The aim of this work is to compare the properties of the narrow component of the iron $\mathrm{K} \alpha$ line with those of the torus obtained by recent MIR studies. The paper is organised as follows. In Sect. 2, we present our MIR/X-ray AGN sample and describe the XMM-Newton/EPIC data analysis; in Sect. 3 we illustrate the $\mathrm{X}$-ray spectral analysis; in Sect. 4 we discuss how to remove the effects of degeneracy caused by the X-ray photon index, the observing angle, the half-opening angle of the torus, and the torus equatorial column density on the values of $\mathrm{Fe} \mathrm{K} \alpha \mathrm{EW}$; and in Sect. 5 we study the relation between the $\mathrm{Fe} \mathrm{K} \alpha \mathrm{EW}$ and the physical characteristics of the torus. In Sect. 6 we discuss our findings and present our conclusions.

\section{Sample and X-ray data analysis}

To study the relation between the iron $\mathrm{K} \alpha$ line and the properties of the torus obtained from MIR studies, we used the sample reported in the recent work of Elitzur (2012), which includes the works of Mor et al. (2009), Nikutta et al. (2009), Alonso-Herrero et al. (2011), Deo et al. (2011), and Ramos Almeida et al. (2011). All these works used the IR clumpy torus model of Nenkova et al. (2002, 2008a,b), which allows fundamental characteristics of the torus to be deduced by fitting MIR spectra. In the model, the optical luminosity is used to estimate the bolometric emission of the accreting system irradiating the torus. The free parameters of this model are the torus width parameter ( $\sigma_{\text {tor }}=\pi / 2-\theta_{\mathrm{OA}}$, see Fig. 1 ), the mean number of clouds along the equatorial line $\left(N_{0}\right)$, the $5500 \AA$ dust optical depth of a single cloud $\left(\tau_{\mathrm{V}}\right)$, the inclination angle of the torus with respect to the line of sight $\left(\theta_{\mathrm{i}}\right)$, the ratio between the outer and inner radius of the torus $(Y)$, and the index of the radial power-law distribution of clouds ( $\mathrm{q}$, where the number of clouds is given by $N(r) \propto r^{-\mathrm{q}}$ ).

We cross-correlated the sample of Elitzur (2012) with the XMM-Newton (Jansen et al. 2001) public data archive (as of November 2012), selecting only type-I AGN to avoid uncertainties in the estimates of the $\mathrm{Fe} \mathrm{K} \alpha$ and continuum flux due to absorption. Amongst the sources with public XMM-Newton observations, PG $1700+518$ was detected with a very low signalto-noise ratio $(\mathrm{S} / \mathrm{N})$, which did not allow constraining the parameters of the $\mathrm{Fe} \mathrm{K} \alpha$ line, so that its spectrum was not used for our study. The final sample contains a total of 49 observations of 24 objects. Most of the sources (19) in our final sample are from the work of Mor et al. (2009), while four sources are taken from Alonso-Herrero et al. (2011) and only one from Ramos Almeida et al. (2011). None of the sources reported in the works of Nikutta et al. (2009) and Deo et al. (2011) were observed by XMM-Newton. Although all the works use the clumpy 


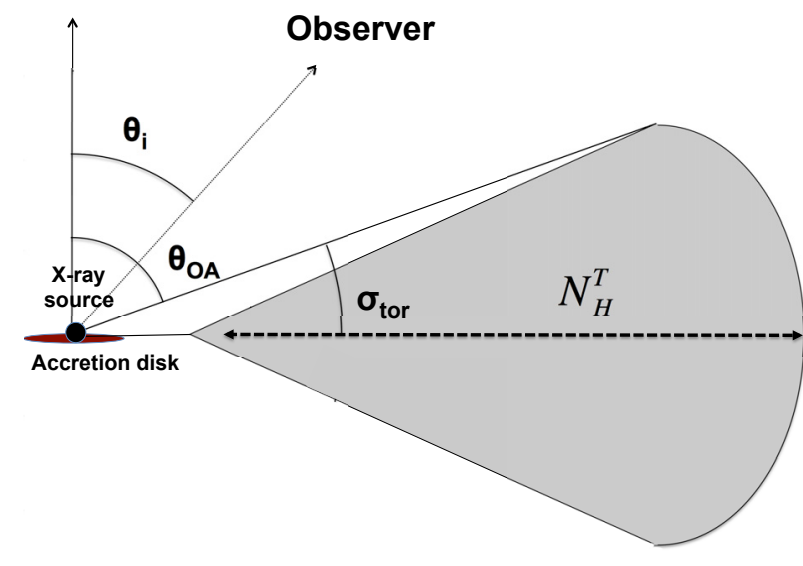

Fig. 1. Schematic representation of the angles considered. $\theta_{\mathrm{i}}$ is the inclination angle of the observer, while $\theta_{\mathrm{OA}}\left(\pi / 2-\sigma_{\text {tor }}\right)$ is the half-opening angle of the torus. $N_{\mathrm{H}}^{\mathrm{T}}$ is the equatorial column density of the torus, i.e. the maximum value of $N_{\mathrm{H}}$ for any value of $\theta_{\mathrm{i}}$.

torus model of Nenkova et al. (2002, 2008a,b), some differences exist in the approach they followed. Mor et al. (2009) fitted the Spitzer/IRS $\sim 2-35 \mu \mathrm{m}$ spectra using a three-component model, which includes a dusty clumpy torus, a clumpy narrowline region (NLR), and black-body emission from hot dust. This last component accounts for the near-IR (NIR, $\lambda \lesssim 5 \mu \mathrm{m}$ ) excess observed when fitting the spectra using only the first two components. In a recent work Mor \& Trakhtenbrot (2011) studied a large sample of $\sim 15000$ AGN and show that most AGN need this hot dust component to explain their NIR spectra. Alonso-Herrero et al. (2011) combined the IR photometric SED with MIR ground-based spectroscopic data in the $8-13 \mu \mathrm{m}$, while Ramos Almeida et al. (2011) only used photometric data. Both Alonso-Herrero et al. (2011) and Ramos Almeida et al. (2011) fitted the data using only the clumpy torus model, because the high angular resolution data they use in their work allows contamination from NLR dust to be ignored.

To study the Fe $\mathrm{K} \alpha$ EW we used the data obtained by the PN (Strüder et al. 2001) and MOS (Turner et al. 2001) cameras on-board XMM-Newton. The original data files (ODFs) were downloaded from the XMM-Newton Science Archive (XSA) ${ }^{1}$ and then reduced using the XMM-Newton Standard Analysis Software (SAS) version 12.0.1 (Gabriel et al. 2004). The raw $\mathrm{PN}$ and MOS data files were processed using the epchain and emchain tasks, respectively. For each observation we checked the background light curve in the $10-12 \mathrm{keV}$ energy band of the data sets in order to detect and filter the exposures for periods of high background activity. We selected only patterns that correspond to single and double events (PATTERN $\leq 4$ ) for PN, and to single, double, triple, and quadruple events for MOS (PATTERN $\leq 12$ ), as suggested by the standard guidelines. The source spectra were extracted from the final filtered event list using circular regions centred on the object (with a typical radius of 30 arcsec), while the background was estimated from regions close to the source (preferably on the same CCD), where no other source was present (with a radius of $40 \mathrm{arcsec}$ ). For sources detected with a low $\mathrm{S} / \mathrm{N}$, we extracted the spectra using a smaller radius ( 10 arcsec). We checked for pile-up with the epatplot task, and for those observations where it was significant (see Table 1), we used annular regions centred on the source, with an inner radius of 5 to 15 arcsec, depending on the strength of the pile-up. We added a multiplicative factor to the models

\footnotetext{
1 http://xmm.esac.esa.int/xsa/
}

to account for cross-calibration between PN and MOS. We fixed the factor to 1 for EPIC/PN and left the MOS1 and MOS2 factors free. For all the spectra the value of the factor turned out to be close to one within a few percentage points. The ancillary response matrices (ARFs) and the detector response matrices (RMFs) were generated using the tasks arfgen and rmfgen, respectively. The spectra were grouped to have at least 20 counts per bin, in order to use $\chi^{2}$ statistics.

The list of AGN used, together with the values of their redshift $(z)$, of the Galactic column density in their direction $\left(N_{\mathrm{H}}^{\mathrm{G}}\right)$, and their X-ray observation log is reported in Table 1.

\section{X-ray spectral analysis}

The X-ray spectral analysis was carried out using XSPEC 12.7.1b (Arnaud 1996). Since we are dealing with objects that may have different characteristics in the X-rays, we started the analysis from a simple baseline model and then added absorbing or emitting components to improve the $\chi^{2}$. More complex models were adopted based on the results of the F-test, using a probability of $p=95 \%$ as a threshold. The baseline model consists of a power-law continuum absorbed by Galactic absorption plus a Gaussian line to account for the iron $\mathrm{K} \alpha$ emission ( $\mathrm{wa}_{\mathrm{G}}$ *(zpo + zgauss) in XSPEC).

The most common features observed in the X-ray spectra of type-I AGN are ionised absorbers (often called warm absorbers) and a soft excess. Ionised absorption is believed to be produced in disk outflows (e.g. Turner \& Miller 2009) and was accounted for using the zxipcf model (Reeves et al. 2008). This multiplicative model uses a grid of XSTAR (Kallman \& Bautista 2001; Bautista \& Kallman 2001) photoionised absorption models, and its free parameters are the covering factor of the ionised absorber $f_{\mathrm{W}}$, its column density $\left(N_{\mathrm{H}}^{\mathrm{W}}\right)$ and its ionisation parameter $(\xi)$. The ionisation parameter is given by $\xi=L_{\text {ion }} / n r^{2}$, where $L_{\text {ion }}$ and $r$ are the $5 \mathrm{eV}-300 \mathrm{keV}$ luminosity and distance from the absorber of the ionising source, respectively, while $n$ is the density of the absorber. The origin of the soft excess is still controversial and might be related to blurred reflection (e.g. Crummy et al. 2006), to Comptonisation of ultraviolet disk photons in a plasma cooler than the one responsible for the primary continuum (e.g. Mehdipour et al. 2011; Noda et al. 2013), or to smeared absorption (e.g. Gierliński \& Done 2004). Since we are not interested in a detailed analysis of the soft excess, we adopted a simple phenomenological model (bremsstrahlung) to account for this feature. All the sources of the sample require more complex models than the baseline. The models we applied to fit the X-ray spectra are the following (listed in the order in which they were applied):

Model A. Baseline model and a bremsstrahlung component at low energies to represent the soft excess. In XSPEC this is written as $\mathrm{wa}_{\mathrm{G}} *(\mathrm{zpo}+\mathrm{bremss}+\mathrm{zgauss})$. The free parameters of this model are the photon index of the power-law continuum $(\Gamma)$, the temperature of the bremsstrahlung $(\mathrm{kT})$, the energy of the Fe $\mathrm{K} \alpha$ line $\left(E_{\mathrm{K} \alpha}\right)$, and the normalisations of the three components. This model was used for 19 observations.

Model B. Baseline model absorbed by a partially covering warm absorber: $\mathrm{wa}_{\mathrm{G}}{ }^{*} \mathrm{zxipcf}$ (zpo + zgauss). The free parameters are those of the baseline model, plus the parameters of the warm absorber $\left(\xi, N_{\mathrm{H}}^{\mathrm{W}}\right.$ and $\left.f_{\mathrm{W}}\right)$. This model was adopted for three observations. 
Table 1. XMM-Newton observation log.

\begin{tabular}{|c|c|c|c|c|c|c|c|}
\hline Source & $\begin{array}{l}(1) \\
N\end{array}$ & $\begin{array}{c}(2) \\
N_{\mathrm{H}}^{\mathrm{G}} \\
{\left[10^{20} \mathrm{~cm}^{-2}\right]}\end{array}$ & $\begin{array}{l}\text { (3) } \\
z\end{array}$ & $\begin{array}{c}(4) \\
\text { Obs. mode/filter }\end{array}$ & $\begin{array}{c}(5) \\
\text { Obs. date } \\
\text { YYYY-MM-DD }\end{array}$ & $\begin{array}{c}(6) \\
\text { Obs. ID }\end{array}$ & $\begin{array}{c}(7) \\
\text { Net exposure } \\
{[\mathrm{ks}]}\end{array}$ \\
\hline B2 2201+31A & & 11.8 & 0.2980 & $\mathrm{FF} / \mathrm{M} ; \mathrm{FF} / \mathrm{M} ; \mathrm{FF} / \mathrm{M}$ & $2008-12-01$ & 0550871001 & $13.5 / 13.2 / 13.2$ \\
\hline IC 4329A & $\begin{array}{l}\text { I } \\
\text { II }\end{array}$ & 4.42 & 0.0160 & $\begin{array}{c}\mathrm{FF} / \mathrm{M}^{\mathrm{P}} ; \mathrm{LW} / \mathrm{M}^{\mathrm{P}} ; \mathrm{LW} / \mathrm{M}^{\mathrm{P}} \\
\mathrm{SW} / \mathrm{T} ; \mathrm{LW} / \mathrm{M} ; \mathrm{LW} / \mathrm{T}\end{array}$ & $\begin{array}{l}2001-01-31 \\
2003-08-06\end{array}$ & $\begin{array}{l}0101040401 \\
0147440101\end{array}$ & $\begin{array}{c}10.4 / 10.4 / 10.4 \\
118.4 / 118.3 / 118.3\end{array}$ \\
\hline NGC 3227 & $\begin{array}{l}\text { I } \\
\text { II }\end{array}$ & 2.13 & 0.0037 & $\begin{array}{c}\text { FF/M; FF/M; FF/M } \\
\text { LW/M; SW/M; SW/M }\end{array}$ & $\begin{array}{l}2000-11-29 \\
2006-12-03\end{array}$ & $\begin{array}{l}0101040301 \\
0400270101\end{array}$ & $\begin{array}{c}34.6 / 34.4 / 34.4 \\
101.3 / 101.0 / 101.0\end{array}$ \\
\hline NGC 4151 & $\begin{array}{l}\text { I } \\
\text { II } \\
\text { III } \\
\text { IV } \\
\text { V } \\
\text { VI } \\
\text { VII } \\
\text { VIII }\end{array}$ & 1.99 & 0.0033 & $\begin{array}{c}\text { FF/M; FF/M } / M^{\mathrm{P}} ; \mathrm{FF} / \mathrm{M}^{\mathrm{P}} \\
\text { SW/M; SW/M; SW/M } \\
\text { FF/M; FF/M; FF/M } \\
\text { SW/M; SW/M } / M^{\mathrm{P}} ; \mathrm{SW} / \mathrm{M}^{\mathrm{P}} \\
\text { SW/M; SW/M } ; \text { SW/MP/M } \\
\text { SW/M; SW/M } ; \text { SW/M } \\
\text { SW/M; SW/M; SW/M } \\
\text { SW/M; SW/M; SW/M }\end{array}$ & $\begin{array}{l}2000-12-22 \\
2000-12-22 \\
2000-12-22 \\
2003-05-25 \\
2003-05-27 \\
2003-05-27 \\
2006-05-16 \\
2006-11-30\end{array}$ & $\begin{array}{l}0112830201 \\
0112310101 \\
0112830501 \\
0143500101 \\
0143500201 \\
0143500301 \\
0402660101 \\
0402660201\end{array}$ & $\begin{array}{l}57.0 / 56.9 / 56.9 \\
29.9 / 29.3 / 29.3 \\
19.7 / 19.6 / 19 / 6 \\
18.5 / 18.3 / 18.4 \\
18.4 / 18.1 / 18.1 \\
18.5 / 18.0 / 18.0 \\
40.0 / 39.8 / 39.8 \\
46.5 / 37.5 / 37.4\end{array}$ \\
\hline NGC 6814 & & 12.8 & 0.0052 & $\mathrm{FF} / \mathrm{M}^{\mathrm{P}} ; \mathrm{FF} / \mathrm{M}^{\mathrm{P}} ; \mathrm{FF} / \mathrm{M}^{\mathrm{P}}$ & $2009-04-22$ & 0550451801 & $28.4 / 28.4 / 28.4$ \\
\hline NGC 7469 & $\begin{array}{l}\text { I } \\
\text { II } \\
\text { III } \\
\text { IV }\end{array}$ & 4.86 & 0.0159 & $\begin{array}{c}\mathrm{SW} / \mathrm{M} ; \mathrm{LW} / \mathrm{M}^{\mathrm{P}} ; \mathrm{FF} / \mathrm{M}^{\mathrm{P}} \\
\mathrm{SW} / \mathrm{M} ; \mathrm{LW} / \mathrm{M} ; \mathrm{FF} / \mathrm{M} \\
\mathrm{SW} / \mathrm{M} ;-;- \\
\mathrm{SW} / \mathrm{M} ;-;-\end{array}$ & $\begin{array}{l}2000-12-26 \\
2000-12-26 \\
2004-11-30 \\
2004-12-03\end{array}$ & $\begin{array}{l}0112170101 \\
0112170301 \\
0207090101 \\
0207090201\end{array}$ & $\begin{array}{c}17.6 / 17.1 / 17.1 \\
23.1 / 22.6 / 22.6 \\
84.5 /-/- \\
78.6 /-/-\end{array}$ \\
\hline PG $0050+124$ & $\begin{array}{l}\text { I } \\
\text { II }\end{array}$ & 5.00 & 0.0609 & $\begin{array}{c}\mathrm{LW} / \mathrm{M}^{\mathrm{P}} ; \mathrm{SW} / \mathrm{M}^{\mathrm{P}} ; \mathrm{SW} / \mathrm{M}^{\mathrm{P}} \\
\mathrm{SW} / \mathrm{M} ; \mathrm{SW} / \mathrm{M} ; \mathrm{SW} / \mathrm{M}^{2}\end{array}$ & $\begin{array}{l}2002-06-22 \\
2005-07-18\end{array}$ & $\begin{array}{l}0110890301 \\
0300470101\end{array}$ & $\begin{array}{l}19.3 / 19.2 / 19.2 \\
77.6 / 77.3 / 77.3\end{array}$ \\
\hline PG 0157+001 & & 2.59 & 0.1628 & FF/M; LW/M; LW/M & $2000-07-29$ & 0101640201 & 7.5/7.5/7.5 \\
\hline PG 0838+770 & & 2.09 & 0.1313 & FF/M; FF/M; FF/M & 2009-03-02 & 0550870401 & $17.6 / 17.5 / 17.5$ \\
\hline PG $0953+414$ & & 1.14 & 0.2390 & LW/T; LW/T; LW/T & $2001-11-22$ & 0111290201 & $11.3 / 11.3 / 11.3$ \\
\hline PG $1004+130$ & & 3.70 & 0.2406 & FF/M; FF/M; FF/M & 2003-05-04 & 0140550601 & $20.4 / 20.2 / 20.2$ \\
\hline PG $1116+215$ & $\begin{array}{l}\text { I } \\
\text { II } \\
\text { III } \\
\text { IV } \\
\text { V } \\
\text { VI }\end{array}$ & 1.28 & 0.1765 & $\begin{array}{l}\text { LW/T; LW/T; LW/T } \\
\text { SW/T; SW/T; SW/T } \\
\text { SW/T; SW/T; SW/T } \\
\text { SW/T; SW/T; SW/T } \\
\text { SW/T; SW/T; SW/T } \\
\text { SW/T; SW/T; SW/T }\end{array}$ & $\begin{array}{l}2001-12-02 \\
2004-12-17 \\
2004-12-19 \\
2008-05-27 \\
2008-12-15 \\
2008-12-20\end{array}$ & $\begin{array}{l}0111290401 \\
0201940101 \\
0201940201 \\
0554380101 \\
0554380201 \\
0554380301\end{array}$ & $\begin{array}{c}7.0 / 6.0 / 6.0 \\
98.1 / 86.3 / 86.3 \\
7.2 / 6.8 / 6.8 \\
85.7 / 84.0 / 84.0 \\
88.1 / 71.2 / 87.9 \\
87.6 / 79.4 / 79.4\end{array}$ \\
\hline PG 1126-041 & $\begin{array}{l}\text { I } \\
\text { II } \\
\text { III } \\
\text { IV }\end{array}$ & 4.30 & 0.0600 & $\begin{array}{l}\text { LW/T; LW/T; LW/T } \\
\text { FF/M; FF/M; FF/M } \\
\text { FF/M; FF/M; FF/M } \\
\text { FF/M; FF/M; FF/M }\end{array}$ & $\begin{array}{l}2004-12-31 \\
2008-06-15 \\
2008-12-13 \\
2009-06-21\end{array}$ & $\begin{array}{l}0202060201 \\
0556230701 \\
0556231201 \\
0606150101\end{array}$ & $\begin{array}{c}31.3 / 31.0 / 31.0 \\
15.0 / 15.0 / 15.0 \\
5.0 / 5.0 / 5.0 \\
101.0 / 100.9 / 100.9\end{array}$ \\
\hline PG 1229+204 & & 2.21 & 0.0637 & SW/M; FF/M; LW/M & 2005-07-09 & 0301450201 & $24.3 / 24.3 / 24.3$ \\
\hline PG $1244+026$ & & 1.75 & 0.0482 & $\mathrm{FF} / \mathrm{T} ; \mathrm{FF} / \mathrm{M}^{\mathrm{P}} ; \mathrm{FF} / \mathrm{M}^{\mathrm{P}}$ & 2001-06-17 & 0051760101 & $6.3 / 6.2 / 6.2$ \\
\hline PG 1309+355 & & 1.03 & 0.1829 & LW/T; LW/T; LW/T & 2002-06-10 & 0109080201 & $25.3 / 25.3 / 25.3$ \\
\hline PG $1411+442$ & & 1.15 & 0.0896 & $\mathrm{FF} / \mathrm{T} ; \mathrm{FF} / \mathrm{M} ; \mathrm{FF} / \mathrm{M}$ & 2002-07-10 & 0103660101 & $25.0 / 24.8 / 24.8$ \\
\hline PG $1426+015$ & & 2.85 & 0.0860 & $\mathrm{FF} / \mathrm{T}^{\mathrm{P}} ; \mathrm{LW} / \mathrm{T}^{\mathrm{P}} ; \mathrm{LW} / \mathrm{TK}^{\mathrm{P}}$ & $2000-07-28$ & 0102040501 & $6.0 / 4.0 / 4.0$ \\
\hline PG 1435-067 & & 5.34 & 0.1290 & $\mathrm{FF} / \mathrm{M} ; \mathrm{FF} / \mathrm{M} ; \mathrm{FF} / \mathrm{M}$ & 2009-02-02 & 0550870201 & $14.2 / 14.2 / 14.2$ \\
\hline PG $1440+356$ & $\begin{array}{l}\text { I } \\
\text { II } \\
\text { III } \\
\text { IV }\end{array}$ & 1.03 & 0.0770 & $\begin{array}{l}\text { LW/T; LW/T }{ }^{P} ; \mathrm{LW} / \mathrm{T}^{\mathrm{P}} \\
\text { SW/M; SW/M; SW/M } \\
\text { SW/M; SW/M; SW/M } \\
\text { SW/M; SW/M; SW/M }\end{array}$ & $\begin{array}{l}2001-12-23 \\
2003-01-02 \\
2003-01-04 \\
2003-01-07\end{array}$ & $\begin{array}{l}0107660201 \\
0005010101 \\
0005010201 \\
0005010301\end{array}$ & $\begin{array}{l}26.2 / 26.0 / 26.0 \\
24.6 / 24.4 / 24.4 \\
27.5 / 27.0 / 27.0 \\
26.0 / 25.8 / 25.8\end{array}$ \\
\hline PG $1448+273$ & & 2.44 & 0.0645 & LW/M; LW/M; LW/M & 2003-02-08 & 0152660101 & $19.5 / 19.5 / 19.5$ \\
\hline PG $1613+658$ & $\begin{array}{l}\text { I } \\
\text { II }\end{array}$ & 2.87 & 0.1385 & $\begin{array}{l}\mathrm{FF} / \mathrm{T} ; \mathrm{LW} / \mathrm{T} ; \mathrm{LW} / \mathrm{TK} \\
\mathrm{FF} / \mathrm{T} ; \mathrm{LW} / \mathrm{T} ; \mathrm{LW} / \mathrm{TK}\end{array}$ & $\begin{array}{l}2001-04-13 \\
2001-08-29\end{array}$ & $\begin{array}{l}0102040601 \\
0102041301\end{array}$ & $\begin{array}{l}5.3 / 1.0 / 1.0 \\
2.7 / 2.7 / 2.7\end{array}$ \\
\hline PG $1626+554$ & & 1.96 & 0.1320 & LW/T; LW/T; LW/T & 2002-05-05 & 0109081101 & $6.1 / 5.4 / 5.4$ \\
\hline PG 2214+139 & & 4.96 & 0.0663 & $\mathrm{FF} / \mathrm{T} ; \mathrm{FF} / \mathrm{T} ; \mathrm{FF} / \mathrm{T}$ & $2002-05-12$ & 0103660301 & $25.3 / 25.2 / 25.2$ \\
\hline
\end{tabular}

Notes. (1) The number of the observation; (2) the value of the Galactic column density $\left(N_{\mathrm{H}}^{\mathrm{G}}\right)$ in the direction of the source (from Dickey \& Lockman 1990); (3) the redshift (z); (4) the modes and filters of PN, MOS1 and MOS2 exposures; (5) the date and (6) the ID of the observation; and (7) the net exposure times. ${ }^{\mathrm{P}}$ Piled-up exposure. The modes and filters of EPIC are the following. FF: full frame; LW: large window; SW: small window; T: thin; M: medium; TK: thick. 

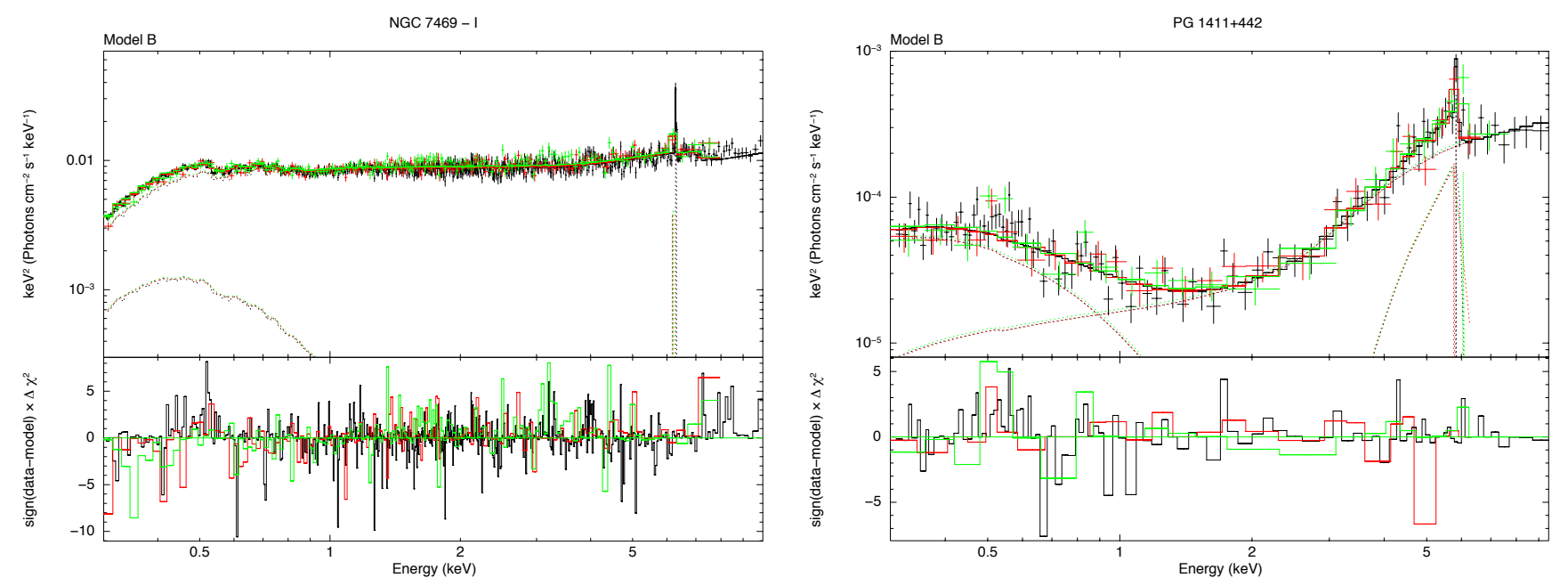

Fig. 2. XMM-Newton EPIC spectra of the first observation of NGC 7469 (left panel) and of PG 1411+442 (right panel). The black points represent EPIC/PN data, and the red and green MOS data. The dotted lines show the different components of the models used. In the case of PG $1411+442$ besides a power law and a narrow $\mathrm{Fe} \mathrm{K} \alpha$ line, a broad relativistic line was added. Details on the models and on their parameters are reported in Appendices A and B.

Model C. Baseline model and a soft excess, absorbed by a partially covering warm absorber: wa $_{\mathrm{G}}{ }^{*} \mathrm{zxipcf}$ (zpo+bremss+zgauss). The free parameters are the same as in model A, plus the parameters of the warm absorber. A total of 18 observations were fitted using this model.

Model $D$. Baseline model absorbed by two partially covering warm absorbers: $w_{G}{ }^{*} z x i p c f * z x i p c f(z p o+z g a u s s)$. The free parameters are the same as in model $\mathrm{B}$, with the addition of the parameters of the second ionised absorber $\left(\xi^{2}, N_{\mathrm{H}, 2}^{\mathrm{W}}, f_{\mathrm{W}}^{2}\right)$. Four observations were fitted using this model.

Model E. Baseline model plus a soft excess and a warm absorber, absorbed by neutral material: $\mathrm{wa}_{\mathrm{G}}{ }^{*} \mathrm{zwabs} * \mathrm{zxipcf}$ (zpo+bremss+zgauss). The free parameters are the same as in model $\mathrm{C}$, plus the column density of the neutral absorber $\left(N_{\mathrm{H}}^{\mathrm{C}}\right)$. This model was used for one observation.

Model F. Baseline model and a soft excess absorbed by two partially covering warm absorbers: $w_{G}{ }^{*} z x i p c f * z x i p c f$ (zpo+bremss + zgauss). The free parameters are the same as in model $\mathrm{D}$, with the addition of the temperature and normalisation of the bremsstrahlung. Three observations were fitted using this model.

Model G. Baseline model and a soft excess, obscured by a neutral and two partially covering ionised absorbers: $\mathrm{wa}_{\mathrm{G}}{ }^{*}$ zwabs*zxipcf*zxipcf(zpo+bremss+zgauss). The free parameters are the same as in model D, with the addition of $N_{\mathrm{H}}^{\mathrm{C}}$ and of the temperature and normalisation of the bremsstrahlung. This model was used for one observation.

For the 14 observations for which it was not possible to constrain the energy of the $\mathrm{Fe} \mathrm{K} \alpha$ line, we fixed the parameter to $E_{\mathrm{K} \alpha}=6.4 \mathrm{keV}$ (in the rest frame of the AGN). We fixed the width of the Gaussian line to $\sigma=1 \mathrm{eV}$, a value below the energy resolution of EPIC/PN and MOS, in order to only consider the narrow core of the iron $\mathrm{K} \alpha$ line. We used the values of the Galactic hydrogen column density $N_{\mathrm{H}}^{\mathrm{G}}$ obtained by Dickey \& Lockman (1990) mapping the HI emission of the Galaxy (see Table 1).

For all the sources, we tested whether adding a broad component of the iron $\mathrm{K} \alpha$ line would significantly improve the fit. This was done using the broad-line profile of Laor (1991) (in XSPEC laor2). Similar to what was done by Nandra et al. (2007), we fixed the internal (for $r \leq R_{\text {break }}$ ) emissivity indices to $\beta_{1}=0$, and the external one (for $r>R_{\text {break }}$ ) to $\beta_{2}=3$. The inclination angle was fixed to the value obtained by MIR studies $\left(i=\theta_{\mathrm{i}}\right)$. We fixed the energy of the broad line to $E_{\mathrm{K} \alpha}^{\mathrm{broad}}=6.4 \mathrm{keV}$ (in the reference frame of the AGN) and tried two scenarios: one in which the inner radius of the iron $\mathrm{K} \alpha$-emitting region is $r_{\text {in }}=6 r_{\mathrm{g}}$ (equivalent to the non-rotating Schwarzschild black hole case), where $r_{\mathrm{g}}=G M_{\mathrm{BH}} / c^{2}$ is the gravitational radius, and the other in which $r_{\text {in }}=1.24 r_{\mathrm{g}}$ (equivalent to the rotating Kerr black hole scenario). The outer radius of the $\mathrm{Fe} \mathrm{K} \alpha$ emitting region was set in both cases to $r_{\text {out }}=400 r_{\mathrm{g}}$, while $R_{\text {break }}$ was left as a free parameter. We performed an F-test using the results obtained with and without relativistic $\mathrm{Fe} \mathrm{K} \alpha$ emission, and rejected the presence of a broad line if the probability was $p<95 \%$. We found that a broad component of the line is needed for 14 observations and 6 objects ( $25 \%$ of the total sample). In the following we use only the narrow component of the $\mathrm{Fe} \mathrm{K} \alpha$ line. For 21 observations additional emission lines (such as O VII, Ne IX, Fe XXV, or Fe XXVI) were needed to obtain a good reduced $\chi^{2}$. In Appendix A we report the values of the main parameters obtained by our spectral analysis, while all the details of the fits are reported in Appendix B. As an example we illustrate in Fig. 2 two typical fits to the X-ray spectra of the sources of our sample. Several of the PG quasars of our sample have been studied by Jiménez-Bailón et al. (2005, see also Piconcelli et al. 2005), and the values of the EW we obtained are consistent with those reported in their paper.

The flux of the power-law continuum in the $2-10 \mathrm{keV}$ band $\left(F_{2-10}\right)$ was obtained using the convolution model cflux in XSPEC. The $k$-corrected continuum luminosities $\left(L_{2-10}\right)$ were calculated using

$L_{2-10}=4 \pi d_{\mathrm{L}}^{2} \frac{F_{2-10}}{(1+z)^{2-\Gamma}}$

where $d_{\mathrm{L}}$ is the luminosity distance. We used standard cosmological parameters $\left(H_{0}=70 \mathrm{~km} \mathrm{~s}^{-1} \mathrm{Mpc}^{-1}, \Omega_{\mathrm{m}}=0.3, \Omega_{\Lambda}=\right.$ $0.7)$. The iron $\mathrm{K} \alpha$ luminosities $\left(L_{\mathrm{K} \alpha}\right)$ were calculated in a similar fashion, excluding the $1 /(1+z)^{2-\Gamma} k$-correction. 


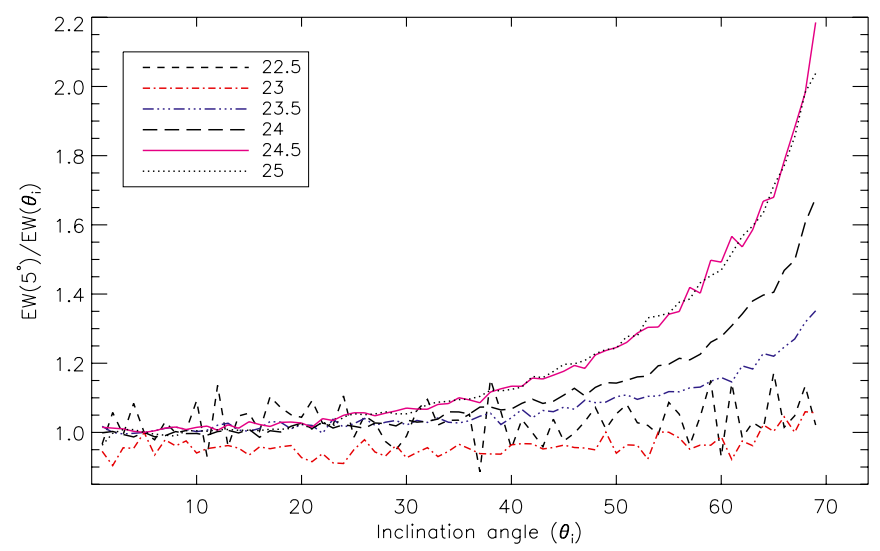

Fig. 3. Ratio of the iron $\mathrm{K} \alpha \mathrm{EW}$ at $\theta_{\mathrm{i}}=5^{\circ}$ and the $E W\left(\theta_{\mathrm{i}}\right)$ for different values of the equatorial column density of the torus $N_{\mathrm{H}}^{\mathrm{T}}$ obtained using the model of Ikeda et al. (2009) for $\theta_{\mathrm{OA}}=70^{\circ}$ and $\Gamma=1.9$. The scatter in the figure is intrinsic to the Monte Carlo simulations.

\section{Renormalising the values of EW}

There are at least six elements that could affect the value of the $\mathrm{Fe} \mathrm{K} \alpha \mathrm{EW}$ and introduce a significant scatter in the correlations with the torus properties obtained by MIR studies: i) variability. The delayed response of the reprocessing material to flux changes of the continuum is expected to have a significant impact on the observed values of EW. ii) The photon index of the $\mathrm{X}$-ray emission. Higher values of $\Gamma$ imply a steeper continuum and fewer photons at the energy of the iron $\mathrm{K} \alpha$ line, which results in lower values of the EW (e.g. Ricci et al. 2013b). iii) The inclination angle of the observer with respect to the torus. For the geometries considered here, lower values of $\theta_{\mathrm{i}}$ produce higher values of EW because the observer is able to see more of the reflected flux (e.g. Ikeda et al. 2009, see Fig. 3). iv) The equatorial column density of the torus $\left(N_{\mathrm{H}}^{\mathrm{T}}\right)$. EW increases with $N_{\mathrm{H}}^{\mathrm{T}}$ up to $\log N_{\mathrm{H}}^{\mathrm{T}} \simeq 24$, and above this value is roughly constant (e.g. Ghisellini et al. 1994). v) The half-opening angle of the torus. The EW of the line decreases for increasing values of $\theta_{\mathrm{OA}}$ (e.g. Ikeda et al. 2009). vi) Metallicity. Lower values of the metallicity produce lower values of EW.

While the impact on the Fe $\mathrm{K} \alpha \mathrm{EW}$ of the first five elements can be reduced, our knowledge of the metallicity of the circumnuclear material of AGN is still poor, so that it is not possible to take this factor into account. In the following, we describe our procedure for renormalising and correcting the values of EW.

\subsection{Variability}

Continuum variability is expected to affect the Fe K $\alpha$ EW measured by single observations of AGN. The bulk of the material responsible for the $\mathrm{Fe} \mathrm{K} \alpha$ line is in fact thought to be located several light years from the X-ray source, so that variations in the continuum do not correspond to simultaneous variations in the line emission. This implies that if a source enters a highflux state, then the flux of the $\mathrm{Fe} \mathrm{K} \alpha$ line relative to that of the continuum (i.e. its $\mathrm{EW}$ ) is lower than the real value, while it would be higher in a low-flux state. This has been confirmed by the recent work of Shu et al. (2012), who found an anticorrelation between EW and flux for different observations of the same sources. Shu et al. (2010) show that variability might also play a role in the X-ray Baldwin effect and that the anticorrelation is attenuated when the values of EW are averaged over several observations. To account for this effect we averaged, when possible, the results obtained by different observations of the same source. Since in several cases the same source was observed at an interval of a few days, we averaged all the parameters obtained by observations carried out within one month. Comparing the fluxes of the narrow $\mathrm{Fe} \mathrm{K} \alpha$ line for the sources for which several observations were available, we found that in almost all cases the fluxes are consistent within the uncertainties. The only exceptions are IC 4329A and NGC 4151. The flux of the narrow line in IC 4329A varies from $1.0_{-0.4}^{+0.3} \times 10^{-12}$ to $5.1_{-0.6}^{+0.8} \times 10^{-13} \mathrm{erg} \mathrm{cm}^{-2} \mathrm{~s}^{-1}$ on a time span of 2.5 years, while in NGC 4151 the line was at its maximum in May 2006 $\left(2.6_{-0.1}^{+0.1} \times 10^{-12} \mathrm{erg} \mathrm{cm}^{-2} \mathrm{~s}^{-1}\right)$ and at its minimum in December $2000\left(1.8_{-0.1}^{+0.1} \times 10^{-12} \mathrm{erg} \mathrm{cm}^{-2} \mathrm{~s}^{-1}\right)$. The low variability of the flux of the narrow $\mathrm{Fe} \mathrm{K} \alpha$ line agrees with the idea that the cold material where the X-ray radiation is reprocessed is located far away from the X-ray source.

\subsection{Removing the dependence on $\Gamma, \theta_{i}$, and $N_{H}^{T}$}

Thanks to the advent of physical torus models such as those developed by Murphy \& Yaqoob (2009; MYTorus ${ }^{2}$ ), Ikeda et al. (2009), and Brightman \& Nandra (2011), it is now possible to reproduce the reflection features produced in the dusty torus surrounding the X-ray source. These models can also be used to remove the dependence of $\mathrm{EW}$ on $\Gamma, \theta_{\mathrm{i}}$, and $N_{\mathrm{H}}^{\mathrm{T}}$, renormalising EW to the same set of values for each source. Removing the degeneracy on EW introduced by these parameters is important to assess the real dependence of EW on the covering factor of the torus (and thus on $\theta_{\mathrm{OA}}$ ). This can be done by using the average values of the photon index obtained by the X-ray spectral fitting $\left(\Gamma^{\text {obs }}\right)$, and the values of the inclination angle $\left(\theta_{i}^{\text {obs }}\right)$ and half-opening angle of the torus $\left(\theta_{\mathrm{OA}}^{\mathrm{obs}}\right)$ obtained by the MIR analysis. The values of the equatorial column density of the torus $\left(N_{\mathrm{H}}^{\mathrm{T}}\right.$,obs $)$ can be extrapolated from the results of the MIR spectral fitting. From the relation between the extinction in the $V$ band and the optical depth $\left(A_{\mathrm{V}}=1.086 \tau_{\mathrm{V}}\right)$, and from the Galactic relation between extinction and column density found by ROSAT $\left(N_{\mathrm{H}}=A_{\mathrm{V}} \cdot 1.79 \times 10^{21} \mathrm{~cm}^{-2}\right.$, Predehl \& Schmitt 1995), for the torus one obtains:

$N_{\mathrm{H}}^{\mathrm{T}, \mathrm{obs}}=1.086 N_{0} \cdot \tau_{\mathrm{V}} \cdot 1.79 \times 10^{21} \mathrm{~cm}^{-2}$.

Equation (2) assumes a Galactic $A_{\mathrm{V}} / N_{\mathrm{H}}$ ratio. This might, however, represent a crude approximation of the real value. Maiolino et al. $(2001 \mathrm{a}, \mathrm{b})$ have shown that the $E(B-V) / N_{\mathrm{H}}$ ratio in $\mathrm{AGN}$ (for $\log L_{2-10} \geq 42$ ) ranges from $\sim 1 \%$ to $\sim 40 \%$ of the Galactic value. As discussed by Maiolino et al. (2001b), this is likely to imply that the $A_{\mathrm{V}} / N_{\mathrm{H}}$ ratio is also significantly lower in AGN than in our Galaxy. To take this effect into account, we used the average value of the $E(B-V) / N_{\mathrm{H}}$ ratio found by Maiolino et al. (2001a) (for $\log L_{2-10} \geq 42$ ) to recalculate the equatorial column density of the torus $\left(N_{\mathrm{H}, \mathrm{Av} \text {. }}^{\mathrm{T}}\right)$. To convert the values of $E(B-V) / N_{\mathrm{H}}$ into $A_{\mathrm{V}} / N_{\mathrm{H}}$, we adopted the Galactic ratio $\left(A_{\mathrm{V}} / E(B-V)=3.1\right)$. The value of $N_{\mathrm{H}, \mathrm{Av}}^{\mathrm{T} \text {. }}$ is is then calculated similarly to what was done in Eq. (2):

$N_{\mathrm{H}, \text { Av. }}^{\mathrm{T}, \mathrm{obs}}=1.086 N_{0} \cdot \tau_{\mathrm{V}} \cdot 1.1 \times 10^{22} \mathrm{~cm}^{-2}$.

In the following we consider both values of $N_{\mathrm{H}}^{\mathrm{T}}$ obtained using Eqs. (2) and (3). The values of $f_{\mathrm{obs}}$ and $N_{\mathrm{H}}^{\mathrm{T}}$ obs are reported in Table A.1.

\footnotetext{
2 http://www .mytorus.com/
} 
Simulations of X-ray absorption and reflection from a clumpy torus have not been carried out yet, and the models listed above consider a smooth dust distribution, different from that of the model of Nenkova et al. (2008a,b). However, assuming that most of the $\mathrm{Fe} \mathrm{K} \alpha$ line is produced in the outer skin of the torus, the differences between the two geometries are likely to be small for type-I AGN. To correct the values of Fe $\mathrm{K} \alpha \mathrm{EW}$, we used the model developed by Ikeda et al. (2009). This model considers a spherical-toroidal geometry for the reprocessing material and has the advantage, with respect to MYTorus, of having the half-opening angle of the torus $\theta_{\mathrm{OA}}$ as a free parameter. The model of Brightman \& Nandra (2011) assumes a similar geometry, but considers a less realistic line-of-sight column density, which is constant for all values of $\theta_{\mathrm{i}}$. The other free parameters of the model of Ikeda et al. (2009) are $\Gamma, N_{\mathrm{H}}^{\mathrm{T}}$, and $\theta_{\mathrm{i}}$. Using the model of Ikeda et al. (2009), we simulated, for each source, a spectrum with the parameters fixed to the values obtained by the observations, and one with a set of parameters arbitrarily chosen $\left(\Gamma=1.9, \theta_{\mathrm{i}}=5^{\circ}, N_{\mathrm{H}}^{\mathrm{T}}=10^{24} \mathrm{~cm}^{-2}\right)$ and with $\theta_{\mathrm{OA}}$ fixed to the value obtained by the fit to the MIR spectra $\left(\theta_{\mathrm{OA}}^{\mathrm{obs}}\right)$. We obtained the value of the Fe $\mathrm{K} \alpha \mathrm{EW}$ of the simulated spectrum ( $\left.E W_{\text {mod }}\right)$ following what was done in Ricci et al. (2013a). For each source we calculated the corrections $K_{\mathrm{I}}$ with

$$
K_{\mathrm{I}}=\frac{E W_{\mathrm{mod}}\left(\Gamma=1.9, \theta_{\mathrm{i}}=5^{\circ}, N_{\mathrm{H}}^{\mathrm{T}}=10^{24} \mathrm{~cm}^{-2}, \theta_{\mathrm{OA}}^{\mathrm{obs}}\right)}{E W_{\mathrm{mod}}\left(\Gamma^{\mathrm{obs}}, \theta_{\mathrm{i}}^{\mathrm{obs}}, N_{\mathrm{H}}^{\mathrm{T}, \mathrm{obs}}, \theta_{\mathrm{OA}}^{\mathrm{obs}}\right)} .
$$

The renormalised equivalent widths $\left(E W_{\text {corr }}^{\mathrm{I}}\right)$ can be easily calculated from the corrections and the observed values of the equivalent width $\left(E W_{\text {obs }}\right)$ :

$E W_{\text {corr }}^{\mathrm{I}}=K_{\mathrm{I}} \times E W_{\mathrm{obs}}$.

As an example of the corrections used, in Fig. 3 we report the trend of $E W\left(5^{\circ}\right) / E W\left(\theta_{\mathrm{i}}\right)$ for different values of $N_{\mathrm{H}}^{\mathrm{T}}$.

\subsection{Removing the dependence on $\Gamma, \theta_{i}$, and $\theta_{O A}$}

To study the intrinsic relation between the $\mathrm{Fe} \mathrm{K} \alpha \mathrm{EW}$ and the equatorial column density of the torus, one can use a procedure similar to the one adopted in Sect. 4.2. Since we are now interested in $N_{\mathrm{H}}^{\mathrm{T}}$, we fixed the half-opening angle to an arbitrary value $\left(\theta_{\mathrm{OA}}=30^{\circ}\right)$ for the reference value of EW and set $N_{\mathrm{H}}^{\mathrm{T}}$ to its observed value. The corrections $K_{\mathrm{II}}$ become

$K_{\mathrm{II}}=\frac{E W\left(\Gamma=1.9, \theta_{\mathrm{i}}=5^{\circ}, N_{\mathrm{H}}^{\mathrm{T}}=N_{\mathrm{H}}^{\mathrm{T}, \mathrm{obs}}, \theta_{\mathrm{OA}}=30^{\circ}\right)}{E W\left(\Gamma^{\mathrm{obs}}, \theta_{\mathrm{i}}^{\mathrm{obs}}, N_{\mathrm{H}}^{\mathrm{T}, \mathrm{obs}}, \theta_{\mathrm{OA}}^{\mathrm{obs}}\right)}$,

while the renormalised equivalent width $\left(E W_{\text {corr }}^{I I}\right)$ can be obtained by

$E W_{\text {corr }}^{\mathrm{II}}=K_{\mathrm{II}} \times E W_{\mathrm{obs}}$.

\section{The relation between the Fe K $\alpha$ EW and the properties of the molecular torus}

\subsection{Covering factor}

As discussed in Mor et al. (2009), the real covering factor of the torus $\left(f_{\text {obs }}\right)$ should be calculated by taking the number of clouds, the half-opening angle of the torus, and the inclination angle of the AGN into account. In the clumpy torus model the probability that the radiation from the central source escapes the torus at a given angle $\beta$ without interacting with the obscuring material is

$P_{\mathrm{esc}}(\beta)=e^{-N_{0} \exp \left(-\frac{\beta^{2}}{\sigma_{\mathrm{tor}}}\right)}$,

where $\beta=\pi / 2-\theta_{\mathrm{i}}$. The geometrical covering factor of the molecular torus is given by integrating $P_{\text {esc }}$ over all angles

$f_{\text {obs }}=1-\int_{0}^{\pi / 2} P_{\mathrm{esc}}(\beta) \cos (\beta) \mathrm{d} \beta$.

If the $\mathrm{Fe} \mathrm{K} \alpha$ line is produced in the torus and the results obtained by applying the clumpy torus model to the MIR spectra of AGN are correct, then a positive correlation between the EW of the line and the real covering factor of the torus would be expected. To study the relation between $\mathrm{Fe} \mathrm{K} \alpha \mathrm{EW}$ and $f_{\text {obs }}$ for our sample, which includes several upper limits, we followed the approach of Guainazzi et al. (2006) and Bianchi et al. (2007), which is an extension of the regression method for left-censoring data described by Schmitt (1985) and Isobe et al. (1986). We performed 10000 Monte-Carlo simulations for each value of the $\mathrm{Fe} \mathrm{K} \alpha \mathrm{EW}$, taking the two following requirements into account: i) the values of EW of the detections were substituted with a random Gaussian distribution, whose mean is given by the value obtained by the fit, and the standard deviation by its error; ii) the upper limits $U$ were substituted with a random uniform distribution in the interval $[0, \mathrm{U}]$. To reduce the degeneracy introduced by different values of $\Gamma, \theta_{\mathrm{i}}$ and $N_{\mathrm{H}}^{\mathrm{T}}$, we used the values of the Fe K $\alpha$ EW corrected as described in Sect. 4.2. For each Monte-Carlo run we fitted the values with a log-linear relationship of the type

$\log E W_{\text {corr }}^{\mathrm{I}}=A+B \cdot f_{\mathrm{obs}}$,

using the ordinary least squares (OLS $[\mathrm{Y} \mid \mathrm{X}]$ ) method. We used the average value of the simulations $(\bar{B})$ as a slope, and as uncertainty their standard deviation. To quantify the significance of the correlation, for each simulation we calculated the Spearman's rank coefficient $(\rho)$ and the null hypothesis probability of the correlation $\left(P_{\mathrm{n}}\right)$, and used the values averaged over all the simulations. Applying Eq. (10), we obtained a slope of $\bar{B}=0.44 \pm$ 0.21 . With the model of Ikeda et al. (2009), it is possible to deduce the expected $E W-f_{\text {obs }}$ trend for the set of parameters we used to renormalise the values of $\mathrm{EW}$. The correct formulation of $f_{\text {obs }}$ is given by Eq. (9), but for $\log N_{\mathrm{H}}^{\mathrm{T}}=24$ at $6.4 \mathrm{keV}$, the escaping probability is $P_{\text {esc }} \sim 0.08$ for $\beta<\sigma_{\text {tor }}$, so that we can approximate the relation to $f_{\mathrm{obs}} \simeq \cos \theta_{\mathrm{OA}}$. We found that for $\Gamma=1.9, N_{\mathrm{H}}^{\mathrm{T}}=10^{24} \mathrm{~cm}^{-2}$, and $\theta_{\mathrm{i}}=5^{\circ}$, the expected slope is $B_{\text {exp }} \simeq 0.4$, consistent with the result of the fit. The scatter plot of $E W_{\text {corr }}^{\mathrm{I}}$ and $E W_{\text {obs }}$ versus $f_{\text {obs }}$ is illustrated in the top panel of Fig. 4. For graphical clarity the data were rebinned to have six values per bin. Performing the statistical tests described above, we found that however the correlation is statistically not significant, with a null hypothesis probability of $P_{\mathrm{n}}=35 \%$ and a Spearman's rank coefficient of $\rho=0.22$. Correcting the values of EW using the equatorial column density of the torus obtained by assuming the average $E(B-V) / N_{\mathrm{H}}$ ratio of Maiolino et al. (2001b) does not alter significantly the results $(\bar{B}=0.46 \pm 0.23$, $\left.\rho=0.23, P_{\mathrm{n}}=37 \%\right)$.

We took random Gaussian errors on $f_{\text {obs }}$ into account, as done for EW, using the errors reported in Alonso-Herrero et al. (2011) and Ramos Almeida et al. (2011) and considering uncertainties of $30 \%$ for the sources of Mor et al. (2009). This does not 


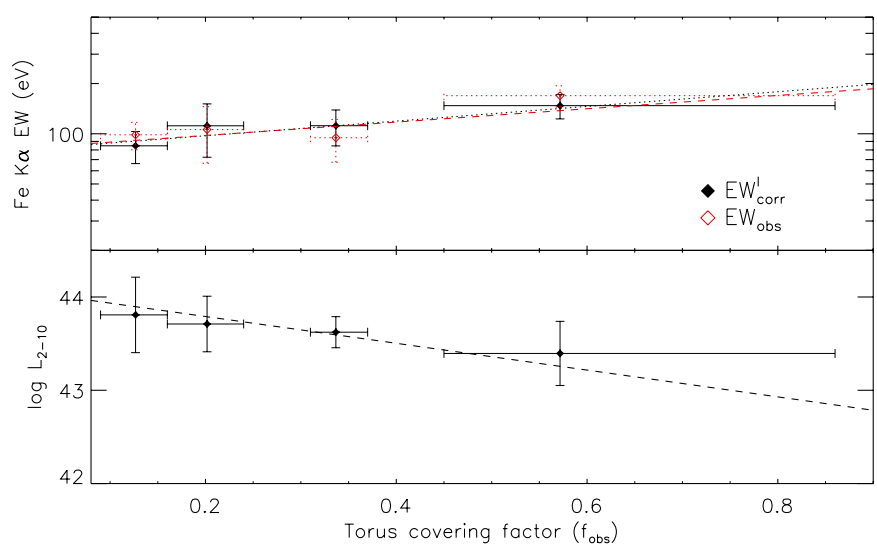

Fig. 4. Top panel: values of the Fe $\mathrm{K} \alpha \mathrm{EW}$ versus the geometrical covering factor of the torus $\left(f_{\text {obs }}\right)$ obtained by fitting MIR spectra with the clumpy torus model. The black filled diamonds are the corrected values (obtained using the model of Ikeda et al. 2009, see Eqs. (4) and (5)), while the empty red ones are the uncorrected values multiplied by an arbitrary constant factor for comparison. The data were rebinned to have six values per bin, and the uncertainties on the Fe K $\alpha$ EW were calculated using the standard error of the mean. The dotted line represents the best fit to the non-binned data obtained by applying Eq. (10), and it has a slope of $\bar{B}=0.44 \pm 0.21$. The red dashed line represents the expected $E W-f_{\text {obs }}$ trend for the set of parameters chosen for the renormalisation, calculated using the model of Ikeda et al. (2009). The intercept of the expected trend was obtained by fitting the data with the slope fixed to the expected value ( $\left.B_{\text {exp }} \simeq 0.4\right)$. Bottom panel: $2-10 \mathrm{keV}$ luminosities versus covering factor of the torus for our sample. The dashed line represents the best fit to the data (Eq. (11).

increase the significance of the correlation, giving a null hypothesis probability of $P_{\mathrm{n}}=38 \%$. We verified whether the fact that the MIR fitting procedures of Mor et al. (2009), Alonso-Herrero et al. (2011), and Ramos Almeida et al. (2011) differ might alter the results. We fitted the data taking only the 19 sources from Mor et al. (2009) into account, and found that the correlation is still not significant $\left(P_{\mathrm{n}}=40 \%\right)$. Consistent results $\left(P_{\mathrm{n}}=34 \%\right.$, $\rho=0.24$ ) were obtained not considering the observations affected by pile-up in the fit.

In the bottom panel of Fig. 4 we show the scatter plot of the $2-10 \mathrm{keV}$ luminosity versus the covering factor of the torus. The two parameters are not significantly correlated $\left(P_{\mathrm{n}}=33 \%\right)$, and by fitting the data we obtained

$\log L_{2-10} \propto(-1.44 \pm 0.78) f_{\text {obs }}$.

\subsection{Equatorial column density of the torus}

With the values of the Fe K $\alpha$ EW corrected to remove the dependence on $\Gamma, \theta_{\mathrm{i}}$, and $\theta_{\mathrm{OA}}$ (Sect. 4.3), we searched for a correlation with the equatorial column density of the torus. Monte Carlo simulations (e.g. Ikeda et al. 2009; Murphy \& Yaqoob 2009) have shown that this parameter is expected to play an important role on the iron $\mathrm{K} \alpha$ line EW. Following the same procedure as discussed in Sect. 5.1, we found that the correlation is statistically not significant $\left(\rho=0.25, P_{\mathrm{n}}=29 \%\right)$. Fitting the data with a log-linear relation of the type

$\log E W_{\text {corr }}^{\mathrm{II}}=\alpha+\beta \cdot \log N_{\mathrm{H}}^{\mathrm{T}}$,

we obtained $\bar{\beta}=0.16 \pm 0.08$. However, due to self-absorption for large values of the column density of the torus, the $\mathrm{Fe} \mathrm{K} \alpha \mathrm{EW}$ is expected to saturate for $\log N_{\mathrm{H}}^{\mathrm{T}} \gtrsim 24$ (e.g. Ghisellini et al. 1994), so that a linear increment is expected only up to this value.

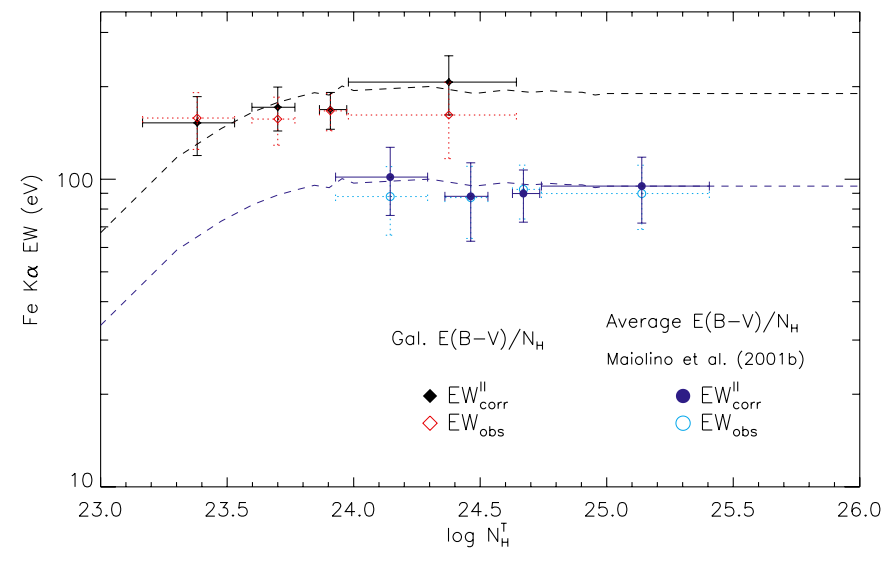

Fig. 5. Values of the $\mathrm{Fe} \mathrm{K} \alpha \mathrm{EW}$ versus the equatorial column density of the torus $\left(N_{\mathrm{H}}^{\mathrm{T}}\right)$ obtained by fitting the MIR spectra. Diamonds and circles represent values of $N_{\mathrm{H}}^{\mathrm{T}}$ obtained using the Galactic $E(B-V) / N_{\mathrm{H}}$ ratio and the average value of Maiolino et al. (2001b), respectively. The black (blue) filled diamonds (circles) are the corrected values (obtained using the model of Ikeda et al. 2009, see Eqs. (6) and (7)), while the empty red (cyan) diamonds (circles) are the uncorrected values. The values of EW were multiplied by an arbitrary constant factor for comparison. The data were rebinned to have six values per bin, and the uncertainties on the $\mathrm{Fe} \mathrm{K} \alpha \mathrm{EW}$ are calculated using the standard error of the mean. The dashed lines represent the expected $E W-N_{\mathrm{H}}^{\mathrm{T}}$ trend for the set of parameters chosen for the renormalisation, calculated using the model of Ikeda et al. (2009). The expected trends were normalised to be compatible with the corrected data.

Considering only the data for $\log N_{\mathrm{H}}^{\mathrm{T}} \leq 24$ resulted in a slope $(\bar{\beta}=0.32 \pm 0.23)$ which is consistent to the expected value $\left(\beta_{\exp }=0.53\right)$ for the same range of $N_{\mathrm{H}}^{\mathrm{T}}$. The trend is, however, statistically non-significant $\left(\rho=0.30, P_{\mathrm{n}}=31 \%\right)$. In Fig. 5 we show the scatter plot of $E W_{\text {corr }}^{\mathrm{II}}$ and $E W_{\mathrm{obs}}$ versus $N_{\mathrm{H}}^{\mathrm{T}}$, together with the expected trend calculated using the model of Ikeda et al. (2009) for the set of parameters used for the re-normalisation. Both the values of $E W_{\text {corr }}^{\mathrm{II}}$ and $E W_{\text {obs }}$ agree with the predicted trend, as would be expected if the line was produced in the molecular torus, although their large associated uncertainties do not allow us to draw a firm conclusion.

\section{Summary and discussion}

Reflection of the power-law continuum from circumnuclear material in AGN is mainly observed through the narrow $\mathrm{Fe} \mathrm{K} \alpha$ line and a reflection hump peaking at $\sim 30 \mathrm{keV}$. The fraction of continuum X-ray flux reflected (hence the $\mathrm{Fe} \mathrm{K} \alpha \mathrm{EW}$ ) is likely to depend strongly on the covering factor of the torus $\left(f_{\text {obs }}\right)$. This implies that $f_{\text {obs }}$, and its evolution with the physical properties of the AGN, is fundamental for a correct understanding of the cosmic X-ray background (CXB, e.g. Gilli et al. 2007). The maximum emission of the CXB is in fact observed at $\sim 30 \mathrm{keV}$ (e.g. Marshall et al. 1980), and a large fraction of CT AGN has often been invoked to correctly reproduce its shape (e.g. Gilli et al. 2007). Because it is observed through a large amount of obscuring material, most of the continuum in these objects is absorbed, which enhances the apparent reflected-to-incident flux ratio, and it makes their observed spectra peak at $\sim 30 \mathrm{keV}$. However, the fraction of Compton-thick sources needed to explain the peak is strongly linked to the fraction of reflected continuum (Gandhi et al. 2007; Treister et al. 2009), and thus to $f_{\text {obs }}$. High values of $f_{\text {obs }}$ have been invoked to explain the characteristics of buried AGN (Ueda et al. 2007; Eguchi et al. 2009, 2011), which 
are type-II objects that have a strong reflection component and a low fraction of scattered continuum. A large covering factor of the torus might explain the strong reflection observed in the hard X-ray spectrum of mildly obscured $\left(23 \leq \log N_{\mathrm{H}}<24\right)$ AGN found by stacking INTEGRAL IBIS/ISGRI data (Ricci et al. 2011) and recently confirmed by Vasudevan et al. (2013) using Swift/BAT. The covering factor of the torus is believed to decrease with luminosity. The original idea of such a relation was put forward by Lawrence \& Elvis (1982) to explain the decrease in the fraction of obscured sources with the luminosity. In the past decade, this trend has been confirmed by several studies carried out at different wavelengths (e.g. Ueda et al. 2003; Beckmann et al. 2009), and it has been shown that it would also be able to straightforwardly explain the X-ray Baldwin effect (Ricci et al. 2013a). Thus a correct understanding of the relation between reflected X-ray radiation and the covering factor of the torus is of the utmost importance for a complete understanding of the X-ray spectral evolution of AGN.

In this work we have studied the relation between the $\mathrm{Fe} \mathrm{K} \alpha \mathrm{EW}$ and important physical properties of the molecular torus, such as its covering factor and equatorial column density $\left(N_{\mathrm{H}}^{\mathrm{T}}\right)$, for a sample of 24 AGN. This was done by combining XMM-Newton/EPIC observations in X-rays with the results obtained by recent MIR spectral studies carried out using the clumpy torus models of Nenkova et al. (2008a,b). The physical torus model of Ikeda et al. (2009) was used to correct the values of the $\mathrm{Fe} \mathrm{K} \alpha \mathrm{EW}$, in order to remove the degeneracy introduced by different values of $\Gamma, \theta_{\mathrm{i}}$, and $N_{\mathrm{H}}^{\mathrm{T}}$. We found that, although the correlation between the $\mathrm{Fe} \mathrm{K} \alpha \mathrm{EW}$ and the covering factor of the torus is statistically non-significant, the slope obtained $(\bar{B}=0.44 \pm 0.21)$ is in very good agreement with the expected value ( $B_{\exp } \simeq 0.4$, see Fig. 4 ). A similar result is obtained when studying the relation between Fe K $\alpha$ EW and $N_{\mathrm{H}}^{\mathrm{T}}$ for $\log N_{\mathrm{H}}^{\mathrm{T}} \leq 24$ : the slope obtained $(\bar{\beta}=0.32 \pm 0.23)$ is consistent with the predicted value $\left(\bar{\beta}_{\exp }=0.53\right.$, see Fig. 5$)$, although the correlation is statistically non-significant.

The fact that the correlation between EW and $f_{\text {obs }}$ is statistically not significant is probably related to the large errors of $\mathrm{Fe} \mathrm{K} \alpha \mathrm{EW}$, and to the large number of PG quasars in the sample, which skews the luminosity distribution towards high values. We also cannot exclude the effect of systematic errors introduced by the technique used to fit the MIR spectra. As argued by Mor et al. (2009), there are two main uncertainties associated to their treatment: variability between the non-simultaneous optical and MIR observations and their choice of the bolometric corrections. These uncertainties could introduce scatter into the torus parameters obtained, so that larger samples, with a more uniform luminosity distribution, are probably needed to find a clear trend between Fe K $\alpha \mathrm{EW}, f_{\mathrm{obs}}$, and $N_{\mathrm{H}}^{\mathrm{T}}$. Another possible source of uncertainty could be introduced by the fact that the model of Nenkova et al. (2008a,b) assumes the inner radius of the torus given by

$R_{\mathrm{d}}=0.4\left(\frac{L_{\mathrm{bol}}}{10^{45} \mathrm{erg} \mathrm{s}^{-1}}\right)^{1 / 2}\left(\frac{1500 \mathrm{~K}}{T_{\text {sub }}}\right) \mathrm{pc}$,

where $L_{\mathrm{bol}}$ is the bolometric luminosity of the AGN and $T_{\text {sub }}$ is the dust sublimation temperature. NIR reverberation studies of the torus have shown that Eq. (13) overestimates the value of $R_{\mathrm{d}}$, which is found to be systematically smaller by a factor of three (Kishimoto et al. 2007). Kawaguchi \& Mori (2010) show that the discrepancy is probably related to the fact that the accretion disk emits anisotropically, so that the effective inner radius is smaller than predicted by Eq. (13). Furthermore, it has been suggested that the observed NIR excess may be due to the fact that silicate and graphite grains sublimate at different temperatures and that large grains are cooling more efficiently, leading to a sublimation zone rather than a sublimation radius (e.g. Kishimoto et al. 2007; Mor \& Netzer 2012). Although the hot graphite-only zone is a plausible source of an additional NIR emission, this component still has to be consistently included in the radiative transfer modelling. Schartmann et al. (2009) models do account for the latter effect by separating the grains of different sizes, but no NIR bump is seen. Other suggestions for the source of the NIR excess include an additional component of low-density interclump dust (Stalevski et al. 2012, 2013) or assume that NIR and MIR emission are coming from two spatially very distinct regions (Hönig et al. 2013). Moreover, modelling of the dusty tori IR emission comes with caveats of its own (Hönig 2013), and model parameters are often degenerate, sometimes resulting in similarly good fits for different combinations of parameters. This inevitably introduces additional uncertainties in any analysis that relies on the properties of the torus obtained from fitting their IR SEDs.

An additional source of scatter might be related to the fact that $\mathrm{Fe} \mathrm{K} \alpha$ emission originating in the torus is subject to a significant contamination from other regions of the AGN, such as the BLR or the outer part of the accretion disk. This degeneracy will be broken in a few years with the advent of ASTRO-H (Takahashi et al. 2010). Thanks to the unprecedented energy resolution in the Fe K $\alpha$ energy band of its X-ray calorimeter (SXS, $5 \mathrm{eV} \mathrm{FWHM}$ at $6 \mathrm{keV}$ ), ASTRO-H will be able to disentangle the emission produced in the torus from that arising from different regions of the AGN.

Acknowledgements. We thank the anonymous referee for his/her comments that helped improve the paper. We thank Chin Shin Chang and Almudena Alonso Herrero for their comments on the manuscript. C.R. is a Fellow of the Japan Society for the Promotion of Science (JSPS). This work was partly supported by the Grant-in-Aid for Scientific Research 23540265 (YU) from the Ministry of Education, Culture, Sports, Science and Technology of Japan (MEXT). P.G. acknowledges support from STFC grant reference ST/J00369711. M.S. acknowledges support of the Ministry of Education, Science and Technological Development of the Republic of Serbia through the projects Astrophysical Spectroscopy of Extragalactic Objects (176001) and Gravitation and the Large Scale Structure of the Universe (176003), and by FONDECYT through grant No. 3140518. This research has made use of the NASA/IPAC Extragalactic Database (NED) which is operated by the Jet Propulsion Laboratory, of data obtained from the High Energy Astrophysics Science Archive Research Center (HEASARC), provided by NASA's Goddard Space Flight Center, and of the SIMBAD Astronomical Database, which is operated by the Centre de Données astronomiques de Strasbourg. Based on observations obtained with $X M M-N e w t o n$, an ESA science mission with instruments and contributions directly funded by ESA Member States and NASA.

\section{References}

Alonso-Herrero, A., Ramos Almeida, C., Mason, R., et al. 2011, ApJ, 736, 82 Antonucci, R. 1993, ARA\&A, 31, 473

Antonucci, R. R. J., \& Miller, J. S. 1985, ApJ, 297, 621

Arnaud, K. A. 1996, in Astronomical Data Analysis Software and Systems V, eds. G. H. Jacoby, \& J. Barnes, ASP Conf. Ser., 101, 17

Baldwin, J. A. 1977, ApJ, 214, 679

Bautista, M. A., \& Kallman, T. R. 2001, ApJS, 134, 139

Beckmann, V., Soldi, S., Ricci, C., et al. 2009, A\&A, 505, 417

Bianchi, S., Guainazzi, M., Matt, G., \& Fonseca Bonilla, N. 2007, A\&A, 467, L19

Bianchi, S., Maiolino, R., \& Risaliti, G. 2012, Adv. Astron., 2012

Brightman, M., \& Nandra, K. 2011, MNRAS, 413, 1206

Crummy, J., Fabian, A. C., Gallo, L., \& Ross, R. R. 2006, MNRAS, 365, 1067

de La Calle Pérez, I., Longinotti, A. L., Guainazzi, M., et al. 2010, A\&A, 524, A50

Deo, R. P., Richards, G. T., Nikutta, R., et al. 2011, ApJ, 729, 108

Dickey, J. M., \& Lockman, F. J. 1990, ARA\&A, 28, 215

Eguchi, S., Ueda, Y., Terashima, Y., Mushotzky, R., \& Tueller, J. 2009, ApJ, 696,1657 
Eguchi, S., Ueda, Y., Awaki, H., et al. 2011, ApJ, 729, 31

Elitzur, M. 2012, ApJ, 747, L33

Fabian, A. C., \& Vaughan, S. 2003, MNRAS, 340, L28

Fukazawa, Y., Hiragi, K., Mizuno, M., et al. 2011, ApJ, 727, 19

Gabriel, C., Denby, M., Fyfe, D. J., et al. 2004, in Astronomical Data Analysis

Software and Systems (ADASS) XIII, eds. F. Ochsenbein, M. G. Allen, \&

D. Egret, ASP Conf. Ser., 314, 759

Gandhi, P., Fabian, A. C., Suebsuwong, T., et al. 2007, MNRAS, 382, 1005

Gandhi, P., Horst, H., Smette, A., et al. 2009, A\&A, 502, 457

Ghisellini, G., Haardt, F., \& Matt, G. 1994, MNRAS, 267, 743

Gierliński, M., \& Done, C. 2004, MNRAS, 349, L7

Gilli, R., Comastri, A., \& Hasinger, G. 2007, A\&A, 463, 79

Guainazzi, M., Siemiginowska, A., Stanghellini, C., et al. 2006, A\&A, 446, 87

Guilbert, P. W., \& Rees, M. J. 1988, MNRAS, 233, 475

Hönig, S. F. 2013, Proc. Torus Workshop, University of Texas, San Antonio, eds. C. Packham, R. Mason, \& A. Alonso-Herrero (University of Texas at San Antonio Libraries), 5-7 December 2012 [arXiv: 1301.1349]

Hönig, S. F., Kishimoto, M., Tristram, K. R. W., et al. 2013, ApJ, 771, 87

Ichikawa, K., Ueda, Y., Terashima, Y., et al. 2012, ApJ, 754, 45

Ikeda, S., Awaki, H., \& Terashima, Y. 2009, ApJ, 692, 608

Isobe, T., Feigelson, E. D., \& Nelson, P. I. 1986, ApJ, 306, 490

Iwasawa, K., \& Taniguchi, Y. 1993, ApJ, 413, L15

Jaffe, W., Meisenheimer, K., Röttgering, H. J. A., et al. 2004, Nature, 429, 47

Jansen, F., Lumb, D., Altieri, B., et al. 2001, A\&A, 365, L1

Jiang, P., Wang, J. X., \& Wang, T. G. 2006, ApJ, 644, 725

Jiménez-Bailón, E., Piconcelli, E., Guainazzi, M., et al. 2005, A\&A, 435, 449

Kallman, T., \& Bautista, M. 2001, ApJS, 133, 221

Kawaguchi, T., \& Mori, M. 2010, ApJ, 724, L183

Kishimoto, M., Hönig, S. F., Beckert, T., \& Weigelt, G. 2007, A\&A, 476, 713

Krolik, J. H., \& Begelman, M. C. 1988, ApJ, 329, 702

Krolik, J. H., Madau, P., \& Zycki, P. T. 1994, ApJ, 420, L57

Laor, A. 1991, ApJ, 376, 90

Lawrence, A., \& Elvis, M. 1982, ApJ, 256, 410

Lightman, A. P., \& White, T. R. 1988, ApJ, 335, 57

Maiolino, R., Marconi, A., \& Oliva, E. 2001a, A\&A, 365, 37

Maiolino, R., Marconi, A., Salvati, M., et al. 2001b, A\&A, 365, 28

Marshall, F. E., Boldt, E. A., Holt, S. S., et al. 1980, ApJ, 235, 4

Mehdipour, M., Branduardi-Raymont, G., Kaastra, J. S., et al. 2011, A\&A, 534, A39

Meisenheimer, K., Tristram, K. R. W., Jaffe, W., et al. 2007, A\&A, 471, 453

Miller, J. S., \& Antonucci, R. R. J. 1983, ApJ, 271, L7

Miyakawa, T., Ebisawa, K., \& Inoue, H. 2012, PASJ, 64, 140

Mor, R., \& Netzer, H. 2012, MNRAS, 420, 526

Mor, R., \& Trakhtenbrot, B. 2011, ApJ, 737, L36

Mor, R., Netzer, H., \& Elitzur, M. 2009, ApJ, 705, 298

Murphy, K. D., \& Yaqoob, T. 2009, MNRAS, 397, 1549

Mushotzky, R. F., Serlemitsos, P. J., Boldt, E. A., Holt, S. S., \& Becker, R. H. 1978, ApJ, 220, 790

Nandra, K., Pounds, K. A., Stewart, G. C., Fabian, A. C., \& Rees, M. J. 1989, MNRAS, 236, 39

Nandra, K., George, I. M., Mushotzky, R. F., Turner, T. J., \& Yaqoob, T. 1997, ApJ, 488, L91

Nandra, K., O’Neill, P. M., George, I. M., \& Reeves, J. N. 2007, MNRAS, 382, 194
Nayakshin, S. 2000, ApJ, 534, 718

Nenkova, M., Ivezić, Ž., \& Elitzur, M. 2002, ApJ, 570, L9

Nenkova, M., Sirocky, M. M., Ivezić, Ž., \& Elitzur, M. 2008a, ApJ, 685, 147

Nenkova, M., Sirocky, M. M., Nikutta, R., Ivezić, Ž., \& Elitzur, M. 2008b, ApJ, 685,160

Nikutta, R., Elitzur, M., \& Lacy, M. 2009, ApJ, 707, 1550

Noda, H., Makishima, K., Nakazawa, K., et al. 2013, PASJ, 65, 4

Page, K. L., O’Brien, P. T., Reeves, J. N., \& Turner, M. J. L. 2004, MNRAS, 347,316

Palmeri, P., Mendoza, C., Kallman, T. R., Bautista, M. A., \& Meléndez, M. 2003, A\&A, 410,359

Piconcelli, E., Jimenez-Bailón, E., Guainazzi, M., et al. 2005, A\&A, 432, 15

Pier, E. A., \& Krolik, J. H. 1992, ApJ, 401, 99

Pier, E. A., \& Krolik, J. H. 1993, ApJ, 418, 673

Pounds, K. A., Nandra, K., Stewart, G. C., \& Leighly, K. 1989, MNRAS, 240, 769

Predehl, P., \& Schmitt, J. H. M. M. 1995, A\&A, 293, 889

Prieto, M. A., Meisenheimer, K., Marco, O., et al. 2004, ApJ, 614, 135

Prieto, M. A., Maciejewski, W., \& Reunanen, J. 2005, AJ, 130, 1472

Raban, D., Jaffe, W., Röttgering, H., Meisenheimer, K., \& Tristram, K. R. W. 2009, MNRAS, 394, 1325

Ramos Almeida, C., Levenson, N. A., Alonso-Herrero, A., et al. 2011, ApJ, 731, 92

Reeves, J., Done, C., Pounds, K., et al. 2008, MNRAS, 385, L108

Ricci, C., Walter, R., Courvoisier, T. J.-L., \& Paltani, S. 2011, A\&A, 532, A102

Ricci, C., Paltani, S., Awaki, H., et al. 2013a, A\&A, 553, A29

Ricci, C., Paltani, S., Ueda, Y., \& Awaki, H. 2013b, MNRAS, 435, 1840

Ricci, C., Ueda, Y., Paltani, S., et al. 2014, MNRAS, 441, 3622

Schartmann, M., Meisenheimer, K., Klahr, H., et al. 2009, MNRAS, 393, 759

Schmitt, J. H. M. M. 1985, ApJ, 293, 178

Shemmer, O., Brandt, W. N., Netzer, H., Maiolino, R., \& Kaspi, S. 2008, ApJ, 682,81

Shu, X. W., Yaqoob, T., \& Wang, J. X. 2010, ApJS, 187, 581

Shu, X. W., Yaqoob, T., \& Wang, J. X. 2011, ApJ, 738, 147

Shu, X. W., Wang, J. X., Yaqoob, T., Jiang, P., \& Zhou, Y. Y. 2012, ApJ, 744, L21

Stalevski, M., Fritz, J., Baes, M., Nakos, T., \& Popović, L. Č. 2012, MNRAS, 420, 2756

Stalevski, M., Fritz, J., Baes, M., \& Popovic, L. C. 2013, Proc. Torus Workshop, University of Texas, San Antonio, eds. C. Packham, R. Mason, \& A. Alonso-Herrero (University of Texas at San Antonio Libraries), 5-7 December 2012 [arXiv: 1301.4244]

Strüder, L., Briel, U., Dennerl, K., et al. 2001, A\&A, 365, L18

Sturm, E., Hasinger, G., Lehmann, I., et al. 2006, ApJ, 642, 81

Takahashi, T., Mitsuda, K., Kelley, R., et al. 2010, in SPIE Conf. Ser., 7732

Treister, E., Urry, C. M., \& Virani, S. 2009, ApJ, 696, 110

Tristram, K. R. W., Meisenheimer, K., Jaffe, W., et al. 2007, A\&A, 474, 837

Turner, T. J., \& Miller, L. 2009, A\&ARv, 17, 47

Turner, M. J. L., Abbey, A., Arnaud, M., et al. 2001, A\&A, 365, L27

Ueda, Y., Akiyama, M., Ohta, K., \& Miyaji, T. 2003, ApJ, 598, 886

Ueda, Y., Eguchi, S., Terashima, Y., et al. 2007, ApJ, 664, L79

Vasudevan, R. V., Mushotzky, R. F., \& Gandhi, P. 2013, ApJ, 770, L37

Yaqoob, T., \& Murphy, K. D. 2011, MNRAS, 412, 1765

Zhou, X.-L., \& Wang, J.-M. 2005, ApJ, 618, L83 


\section{Appendix A: X-ray spectral results}

In Tables A. 1 and A. 2 we report the results of the spectral fitting described in Sect. 3 for our XMM-Newton/EPIC sample.

\section{Appendix B: Notes on the individual sources}

In the following we report the details on the emission lines added to the best fits.

IC $4329 a-I I$. A narrow $(\sigma=1 \mathrm{eV})$ emission feature is detected in the spectrum. The narrow line is found at $E=7.00_{-0.03}^{+0.02} \mathrm{keV}$ $\left(E W=20_{-3}^{+2} \mathrm{eV}\right)$, and is likely to be Fe XXVI.

$N G C 4151$ - I. The spectrum shows evidence of three narrow emission features at low energies. These features were fitted using Gaussian emission lines at $E=0.561_{-0.003}^{+0.003} \mathrm{keV}$ $\left(E W=91_{-4}^{+3} \mathrm{eV}\right), E=0.903_{-0.004}^{+0.003} \mathrm{keV}\left(E W=83_{-3}^{+4} \mathrm{eV}\right)$ and $E=1.33_{-0.01}^{+0.01} \mathrm{keV}\left(E W=37_{-6}^{+3} \mathrm{eV}\right)$. These lines are consistent with O VII, Ne IX, and $\mathrm{Mg} \mathrm{XI}$, respectively.

NGC 4151 - II. Three narrow emission features at low energies are detected. The narrow lines were found to be at $E=$ $0.557_{-0.002}^{+0.004} \mathrm{keV}\left(E W=89_{-2}^{+4} \mathrm{eV}\right), E=0.903_{-0.004}^{+0.003} \mathrm{keV}(E W=$ $\left.78_{-3}^{+4} \mathrm{eV}\right)$, and $E=1.34_{-0.01}^{+0.01} \mathrm{keV}\left(E W=33_{-4}^{+3} \mathrm{eV}\right)$, and they are consistent with $\mathrm{O}$ VII, $\mathrm{Ne}$ IX, and $\mathrm{Mg}$ XI, respectively.

NGC 4151 - III. Three narrow emission features at low energies are detected. The three lines are at $E=0.557_{-0.003}^{+0.004} \mathrm{keV}$ $\left(E W=78_{-4}^{+3} \mathrm{eV}\right), E=0.897_{-0.005}^{+0.007} \mathrm{keV}\left(E W=75_{-3}^{+4} \mathrm{eV}\right)$, and $E=1.31_{-0.02}^{+0.02} \mathrm{keV}\left(E W=37_{-6}^{+4} \mathrm{eV}\right)$, and they are consistent with $\mathrm{O}$ VII, Ne IX, and $\mathrm{Mg} \mathrm{XI}$, respectively.

$N G C 4151$ - IV. Two narrow emission features at low energies are detected. The narrow features are found at $E=$ $0.558_{-0.003}^{+0.003} \mathrm{keV}\left(E W=103_{-1}^{+13} \mathrm{eV}\right)$, and $E=0.897_{-0.005}^{+0.007} \mathrm{keV}$ $\left(E W=76_{-5}^{+4} \mathrm{eV}\right)$, and they are consistent with O VII and Ne IX, respectively.

$N G C 4151-V$. Two narrow emission features at low energies are detected. The narrow features are located at $E=0.558_{-0.002}^{+0.003} \mathrm{keV}$ $\left(E W=108_{-8}^{+7} \mathrm{eV}\right)$, and $E=0.892_{-0.007}^{+0.009} \mathrm{keV}\left(E W=63_{-4}^{+4} \mathrm{eV}\right)$, and they are consistent with O VII and Ne IX, respectively.

$N G C 4151$ - VI. Two narrow emission features at $E=$ $0.559_{-0.002}^{+0.003} \mathrm{keV}\left(E W=100_{-7}^{+13} \mathrm{eV}\right)$ and $E=0.895_{-0.005}^{+0.008} \mathrm{keV}$ $\left(E W=71_{-3}^{+4} \mathrm{eV}\right)$ are detected. The two lines are consistent with $\mathrm{O}$ VII and Ne IX, respectively.

NGC 4151 - VII. Five narrow emission features at low energies are detected. The energies of the narrow lines are $E=0.563_{-0.002}^{+0.002} \mathrm{keV}\left(E W=86_{-3}^{+4} \mathrm{eV}\right), E=0.896_{-0.003}^{+0.004} \mathrm{keV}$ $\left(E W=77_{-3}^{+3} \mathrm{eV}\right), E=1.34_{-0.01}^{+0.01} \mathrm{keV}\left(E W=35_{-3}^{+3} \mathrm{eV}\right)$, $E=1.82_{-0.02}^{+0.02} \mathrm{keV}\left(E W=69_{-5}^{+4} \mathrm{eV}\right)$, and $E=7.04_{-0.07}^{+0.03} \mathrm{keV}$ $\left(E W=32_{-4}^{+5} \mathrm{eV}\right)$. These lines are consistent with being due to O VII, Ne IX, Mg XI, Si XIII, and to Fe XXVI, respectively.
NGC 4151 - VIII. Three narrow emission features at low energies are detected. The narrow lines have energies of $E=0.596_{-0.005}^{+0.005} \mathrm{keV}\left(E W=77_{-2}^{+3} \mathrm{eV}\right), E=0.897_{-0.006}^{+0.006} \mathrm{keV}$ $\left(E W=59_{-3}^{+4} \mathrm{eV}\right)$, and $E=1.78_{-0.02}^{+0.04} \mathrm{keV}\left(E W=50_{-4}^{+5} \mathrm{eV}\right)$, and are consistent with $\mathrm{O}$ VII, Ne IX, and Si XIII, respectively.

$P G 0050+124-I$. The spectrum requires an additional line at $E=6.96_{-0.10}^{+0.09} \mathrm{keV}$, likely due to Fe XXVI, with an equivalent width $E W=106 \pm 24 \mathrm{eV}$.

$P G 0050+124$ - II. Besides the Fe XXVI line at $E=6.97_{-0.04}^{+0.06}$ $\mathrm{keV}(E W=56 \pm 13 \mathrm{eV})$, we found evidence of another unresolved ionised iron line (likely Fe XXV) at $E=6.66_{-0.04}^{+0.05} \mathrm{keV}$ $\left(E W=50_{-10}^{+12} \mathrm{eV}\right)$.

$P G 1116+215-I$. Two lines at $6.7 \mathrm{keV}\left(E W=63_{-34}^{+38} \mathrm{eV}\right)$ and $6.97 \mathrm{keV}\left(E W=128_{-45}^{+49} \mathrm{eV}\right)$ are needed. The lines are consistent with being produced by emission of ionised iron (Fe XXV and Fe XXVI, respectively).

$P G 1116+215-I I I$. Two lines at $6.7 \mathrm{keV}\left(E W=68_{-34}^{+35} \mathrm{eV}\right)$ and $6.97 \mathrm{keV}\left(E W=124_{-45}^{+51} \mathrm{eV}\right)$ are needed. The two lines are consistent with being produced by emission of ionised iron (Fe XXV and Fe XXVI, respectively).

$P G$ 1126-041 - I. Two emission lines at low energies were also needed. The lines are located at $E=0.56 \pm 0.01 \mathrm{keV}(E W=$ $\left.107_{-27}^{+12} \mathrm{eV}\right)$ and $E=0.90 \pm 0.02 \mathrm{keV}\left(E W=68_{-33}^{+37} \mathrm{eV}\right)$, and are consistent with being due to O VII and Ne IX, respectively.

$P G$ 1126-041 - II. Two emission lines at $E=0.60 \pm 0.03 \mathrm{keV}$ $\left(E W=70_{-38}^{+6} \mathrm{eV}\right)$ and $E=0.89 \pm 0.03 \mathrm{keV}(E W \leq 280 \mathrm{eV})$ are also needed. The lines are consistent with being due to O VII and $\mathrm{Ne} I X$, respectively.

$P G$ 1126-041 - III. The spectrum shows an additional emission line at $E=0.60 \pm 0.02 \mathrm{keV}\left(E W=115_{-63}^{+53} \mathrm{eV}\right)$, consistent with the O VII line.

$P G$ 1126-041 - IV. Two lines at low energy were also found. The line at $E=0.58 \pm 0.01 \mathrm{keV}\left(E W=65_{-9}^{+12} \mathrm{eV}\right)$, is likely due to OVII emission, while that at $E=0.93 \pm 0.02 \mathrm{keV}$ $\left(E W=89_{-31}^{+22} \mathrm{eV}\right)$ is consistent with being Ne IX. Another emission line at $E=7.82_{-0.08}^{+0.13} \mathrm{keV}\left(\mathrm{EW}=87_{-40}^{+24} \mathrm{eV}\right)$, consistent with being the $\mathrm{He} \beta$ form of Fe XXV, was also found.

$P G$ 1229+204. An emission line at $E=6.72 \pm 0.05 \mathrm{keV}$ $\left(E W=98_{-25}^{+28} \mathrm{eV}\right)$, consistent with being due to the He $\alpha$ state of $\mathrm{Fe} X X \mathrm{~V}$, is required.

$P G 1440+356-I I$. An emission line at $E=6.72 \pm 0.09 \mathrm{keV}$ $\left(E W=67_{-36}^{+19} \mathrm{eV}\right)$ was detected, and is likely the He $\alpha$ form of Fe XXV.

$P G 1440+356-$ III. The spectrum shows evidence of emission due to the $\mathrm{He} \alpha$ form of Fe XXV at $E=6.73 \pm 0.10 \mathrm{keV}$ $(E W=106 \pm 41 \mathrm{eV})$.

$P G 1440+356-I V$. We found evidence of an emission feature at $E=6.79 \pm 0.07 \mathrm{keV}(E W=197 \pm 48 \mathrm{eV})$, which is probably due to the He $\alpha$ state of Fe XXV. 


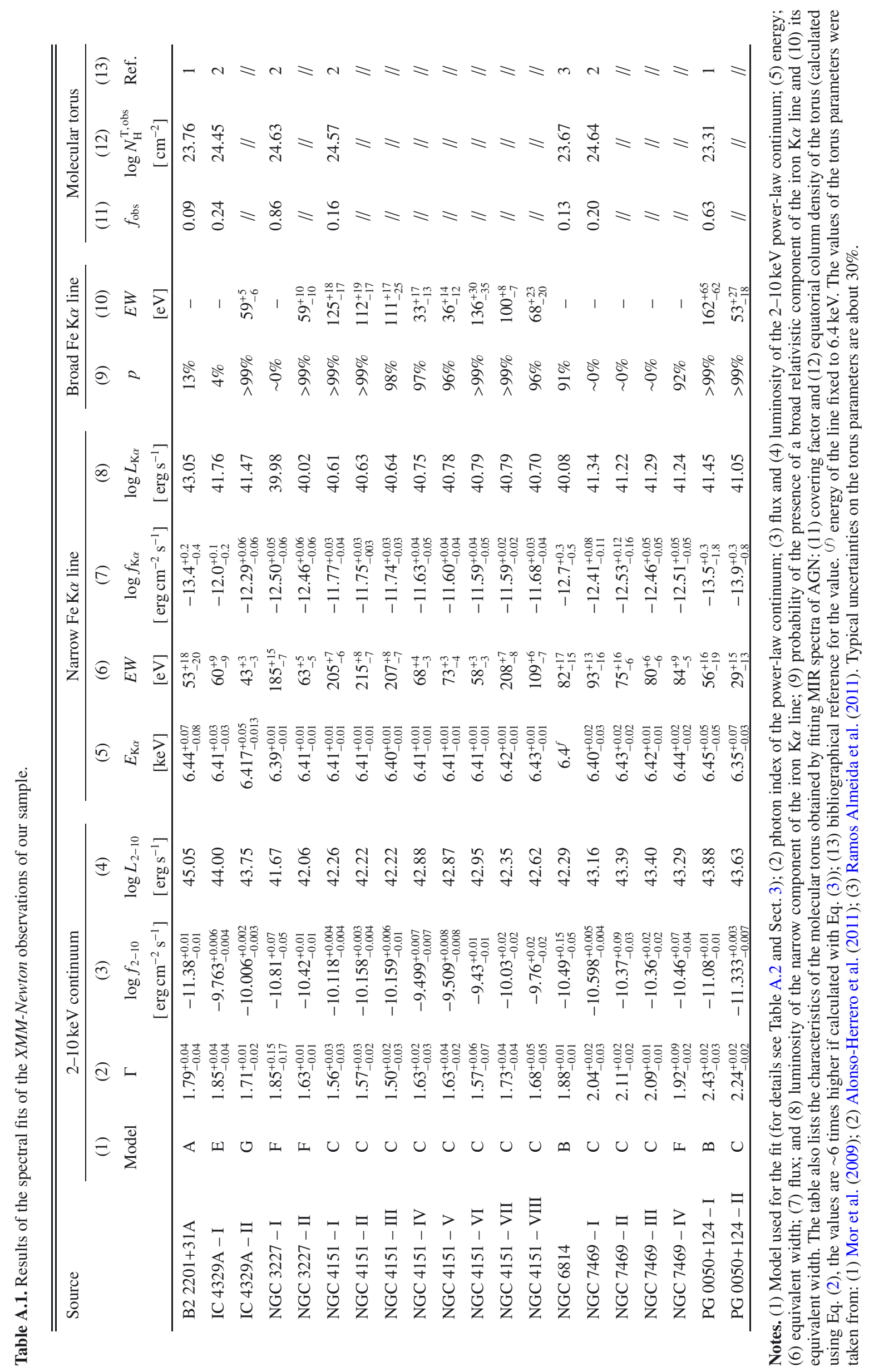


C. Ricci et al.: Iron $\mathrm{K} \alpha$ line and the molecular torus in AGN: an IR/X-ray view

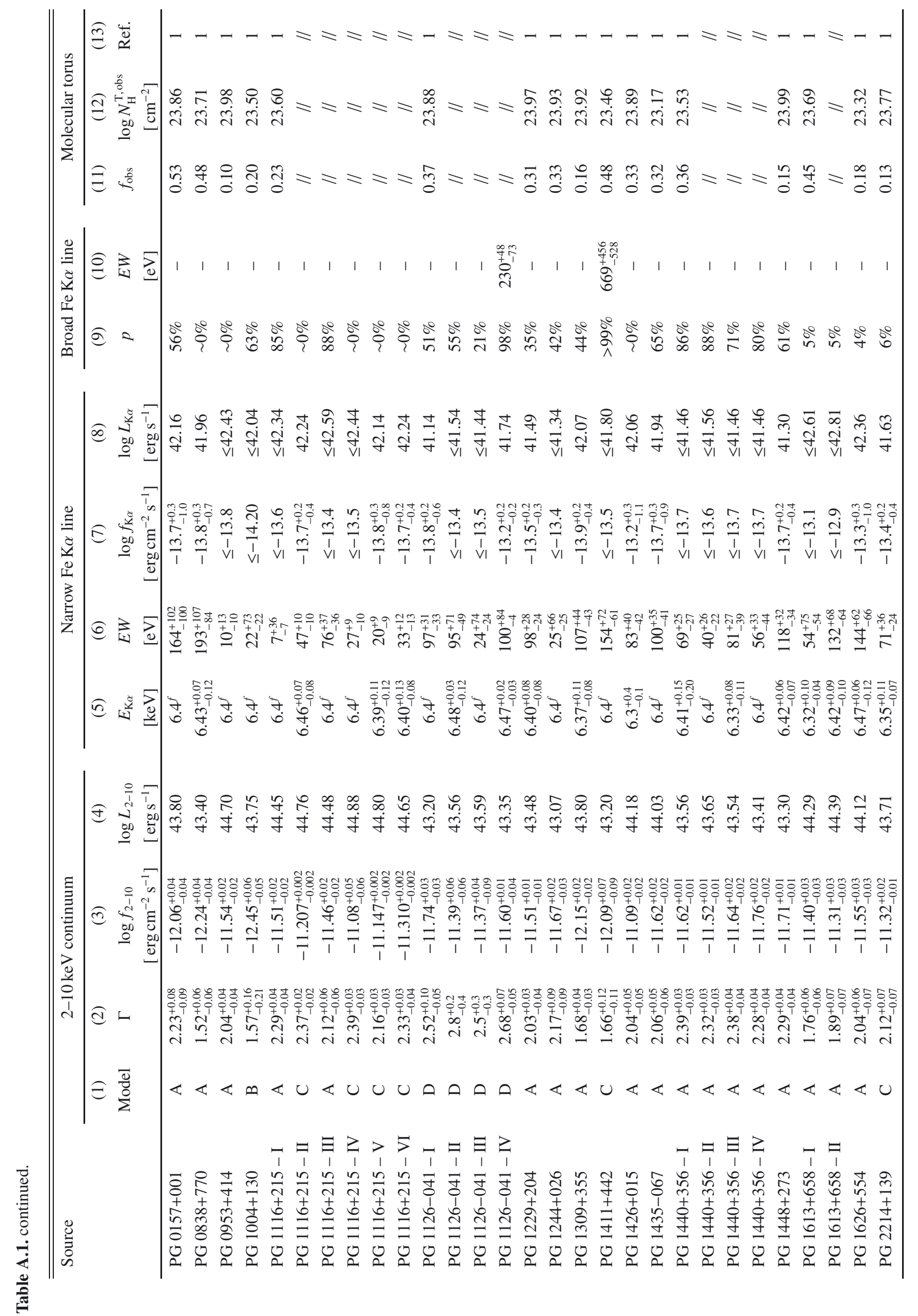




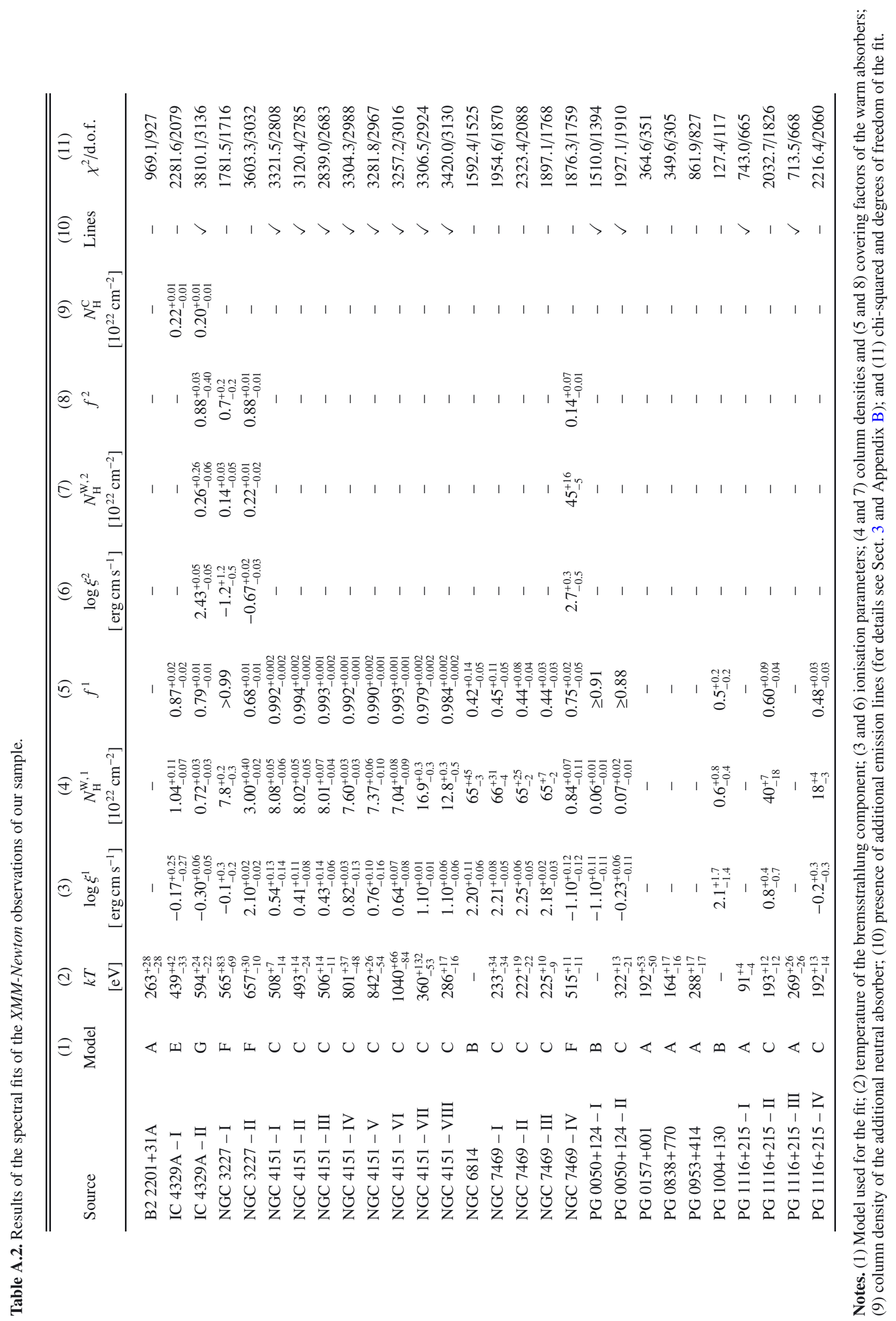




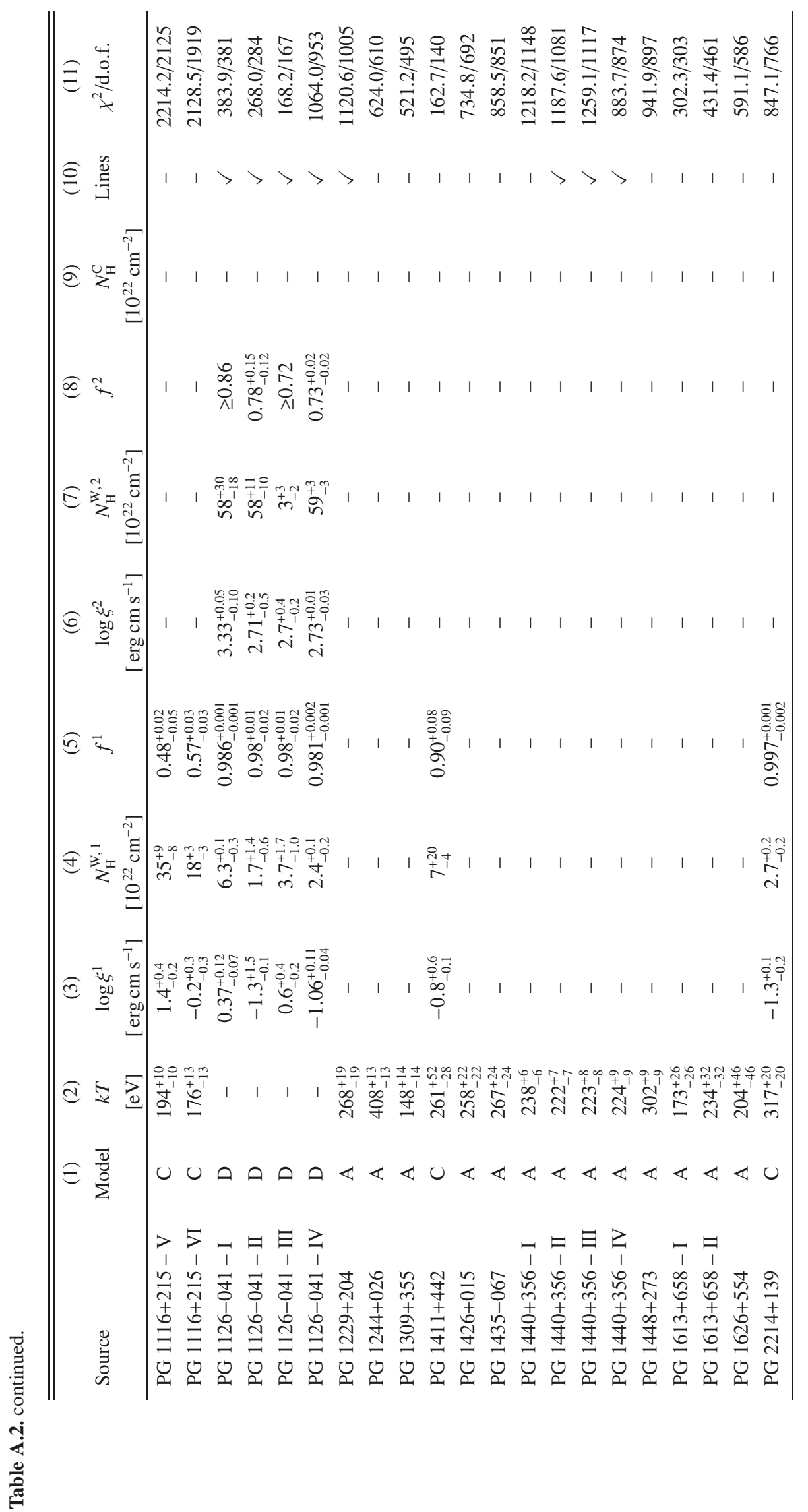

